CAFPE/15-02

LU TP $03-15$

UGFT/145-02

$\frac{\text { hep-ph/0304222 }}{\text { April } 2003}$

\title{
QCD Short-distance Constraints and Hadronic Approximations ${ }^{\dagger}$
}

\author{
Johan Bijnens $^{a}$, Elvira Gámiz ${ }^{b}$, Edisher Lipartia ${ }^{a *}$ and Joaquim Prades ${ }^{b}$ \\ ${ }^{a}$ Department of Theoretical Physics 2, Lund University, \\ Sölvegatan 14A, S 223-62 Lund, Sweden \\ ${ }^{b}$ Centro Andaluz de Física de las Partículas Elementales (CAFPE) and \\ Departamento de Física Teórica y del Cosmos, Universidad de Granada \\ Campus de Fuente Nueva, E-18002 Granada, Spain
}

\begin{abstract}
This paper discusses a general class of ladder resummation inspired hadronic approximations. It is found that this approach naturally reproduces many successes of single meson per channel saturation models (e.g. VMD) and NJL based models. In particular the existence of a constituent quark mass and a gap equation follows naturally. We construct an approximation that satisfies a large set of QCD shortdistance and large $N_{c}$ constraints and reproduces many hadronic observables.

We show how there exists in general a problem between QCD short-distance constraints for Green Functions and those for form factors and cross-sections following from the quark-counting rule. This problem while expected for Green functions that do not vanish in purely perturbative QCD also persists for many Green functions that are order parameters.
\end{abstract}

PACS: 12.38.Lg, 11.15.Pg, 12.39.Fe, 12.39.Ki

\footnotetext{
$\dagger$ Supported in part by the European Union RTN network, Contract No. HPRN-CT-2002-00311 (EURIDICE)

* On leave of absence from Laboratory of Information Technologies, Joint Institute for Nuclear Research, 141980 Dubna, Russia and High Energy Physics Institute, Tbilisi State University, University St. 9, 380086 Tbilisi, Georgia.
} 


\section{Introduction}

Formulating a consistent hadronic approximation to Quantum Chromodynamics (QCD) is an old and very difficult problem. At low energies the solution to this problem is Chiral Perturbation Theory (ChPT) but the domain of validity of this is fairly limited and there tend to be a rather large number of parameters that needs to be dealt with. It cannot be simply extended to the intermediate energy domain. In this paper we describe an approach based on a few simple assumptions and then try to see how far this can go. This fits naturally in the limit of large number of colours $\left(N_{c}\right)$. In this limit and assuming confinement, QCD is known to reduce to a theory of stable hadrons interacting only at tree level [1. So the only singularities in amplitudes are produced by the various tree-level poles occurring. This has long been a problem for various variants of models incorporating some notion of constituent quarks like the Nambu-Jona-Lasinio (NJL) models [2, 3, 4] or the chiral quark model [5].

The main idea in this paper is to take the underlying principle of ladder resummation approaches to hadronic physics and make two successive approximations in this. First we treat the rungs of the ladder as a type of general contact interaction and second the remaining loop-integrations that occur, which are always products of one-loop integrations, we treat as general everywhere analytic functions. The only singularities that occur then are those generated by the resummations and we naturally end up with a hadronic large $N_{c}$ model.

This is also very close to the treatment of the (extended) Nambu-Jona-Lasinio models as given in [6, 7, 8] where $n$-point Green functions ${ }^{1}$ are seen as chains of one-loop bubbles connected by a one-loop with three or more vertices. The one-loop bubbles can be seen as one-loop Green functions as well. The full Green functions there are thus composed of one-loop Green functions glued together by the (ENJL) couplings $g_{V}$ and $g_{S}$. One way to incorporate confinement in these ENJL models is by introducing an infinite number of counterterms to remove all the unwanted singularities 9]. In [9] it was then argued that the ENJL approach was basically identical to a one resonance saturation approach. They then proposed a minimal hadronic ansatz where one resonance saturation is the underlying principle and all couplings should be determined from QCD short-distance and chiral constraints with the relevant short-distance constraints those that result from order parameters. Order parameters are quantities which would be fully zero if only perturbative QCD without quark masses and condensates is considered. This approach has been further discussed for two-point Green functions in [10] and applied to some three-point functions in [11, see also the discussions in [12] for earlier similar uses of order parameters. Problems appear for $n$-point Green functions in that not necessarily all freedom in the parameters can be fixed by the long-distance chiral constraints and/or short-distance constraints or involve too many unknown constants in the chiral constraints.

In this paper we follow a different scheme. We assume that the Green functions are produced by a ladder-resummation like ansatz. They consist of bubble-diagrams put together

\footnotetext{
${ }^{1}$ In the remainder these are often referred to as $n$-point functions.
} 
from one-loop Green functions. We do not use the (constituent) quark-loop expressions for these one-loop Green functions but instead consider them as constants or low-order polynomials in the kinematic variables. This set of assumptions turns out to be rather constraining in the type of model that can be constructed. In particular the gap equation for spontaneous symmetry breaking follows from the requirements of resummation and the full Ward identities as shown in Section 2. The link with constituent quark models is the fact that given the full Ward identities one can define a constituent quark mass, obeying a gap equation, and the one-loop Green functions satisfy the Ward identities with constituent quark-masses. In the two-point function sector this naturally reduces to the approach of [9] but it allows to go beyond two-point functions in a more systematic manner.

In Section 2 we discuss the buildup of the model and the two-point functions. We first work in the chiral limit and then add corrections due to current quark masses. Chiral Perturbation Theory, or low-energy, constraints are naturally satisfied in our approach which is chiral invariant from the start. Also large $N_{c}$ constraints are satisfied naturally. We show how the short-distance constraints can be included. Section 3 treats several threepoint functions and includes here short-distance constraints coming from form factors and from the more suppressed combinations of short-distances.

Numerical results are presented in Section 4. We find a reasonable agreement for the predictions.

Going beyond the one-resonance saturation in this approach is difficult as explained in Section 5. Another point raised is that hadronic models will in general have problems with QCD short-distance constraints, even if the short-distance behaviour is an order parameter, we discuss in detail how the pseudo-scalar-scalar-pseudo-scalar three-point function is a typical example of this problem in Section [6.

We consider this class of models still useful even with the problems inherent in it. They provide a consistent framework to address the problems of nonleptonic matrix-elements where in general very many Green functions with a large number of insertions is needed. The present approach offers a method to analytically calculate these Green functions and thus study the effects of the various ingredients on the final results. One motivation for this work was to understand many of the rather surprising features found in the calculations using the ENJL model of the $B_{K}$ parameter, the $\Delta I=1 / 2$ rule, gluonic and electroweak Penguins, electromagnetic effects and the muon anomalous magnetic moment [13, 14] and improve on those calculations.

The Appendix contains expressions for the short-distance properties of several threepoint functions. 


\section{Basics of the Model and Two-Point Functions}

\subsection{General}

The Lagrangian for the large $N_{c}$ ENJL model is

$$
\begin{aligned}
\mathcal{L}_{E N J L}= & \sum_{i, j, \alpha} \bar{q}_{\alpha}^{i}\left\{\gamma^{\mu}\left(i \partial_{\mu} \delta^{i j}+v_{\mu}^{i j}+a_{\mu}^{i j} \gamma_{5}\right)-\mathcal{M}^{i j}-s^{i j}+i p^{i j} \gamma_{5}\right\} q_{\alpha}^{j} \\
& +2 g_{S} \sum_{i, j, \alpha, \beta}\left(\bar{q}_{R \alpha}^{i} q_{L \alpha}^{j}\right)\left(\bar{q}_{L \beta}^{j} q_{R \beta}^{i}\right) \\
& -g_{V} \sum_{i, j, \alpha, \beta}\left(\bar{q}_{L \alpha}^{i} \gamma_{\mu} q_{L \alpha}^{j}\right)\left(\bar{q}_{L \beta}^{j} \gamma^{\mu} q_{L \beta}^{i}\right)-g_{V} \sum_{i, j, \alpha, \beta}\left(\bar{q}_{R \alpha}^{i} \gamma_{\mu} q_{R \alpha}^{j}\right)\left(\bar{q}_{R \beta}^{j} \gamma^{\mu} q_{R \beta}^{i}\right)
\end{aligned}
$$

with $i, j$ flavour indices, $\alpha, \beta$ colour indices and $q_{R(L)}=(1 / 2)\left(1+(-) \gamma_{5}\right) q$. The flavour matrices $v, a, s, p$ are external fields and can be used to generate all the Green functions we will discuss. The four-quark interactions can be seen as an approximation for the rungs of a ladder-resummation scheme.

The Green functions generated by functional differentiation w.r.t. $v^{i j}(x), a^{i j}(x), s^{i j}(x), p^{i j}(x)$ correspond to Green functions of the currents

$$
\begin{aligned}
V_{\mu}^{i j}(x) & =\bar{q}_{\alpha}^{i}(x) \gamma_{\mu} q_{\alpha}^{j}(x), \\
A_{\mu}^{i j}(x) & =\bar{q}_{\alpha}^{i}(x) \gamma_{\mu} \gamma_{5} q_{\alpha}^{j}(x), \\
S^{i j}(x) & =-\bar{q}_{\alpha}^{i}(x) q_{\alpha}^{j}(x) \\
P^{i j}(x) & =\bar{q}_{\alpha}^{i}(x) i \gamma_{5} q_{\alpha}^{j}(x) .
\end{aligned}
$$

An underlying assumption is that these currents can be identified with the QCD ones.

In the remainder of this section we will discuss the two-point functions

$$
\begin{aligned}
\Pi_{\mu \nu}^{V}(q)^{i j k l} & =i \int d^{d} x e^{i q \cdot x}\left\langle 0\left|T\left(V_{\mu}^{i j}(x) V_{\nu}^{k l}(0)\right)\right| 0\right\rangle, \\
\Pi_{\mu \nu}^{A}(q)^{i j k l} & =i \int d^{d} x e^{i q \cdot x}\left\langle 0\left|T\left(A_{\mu}^{i j}(x) A_{\nu}^{k l}(0)\right)\right| 0\right\rangle, \\
\Pi_{\mu}^{S}(q)^{i j k l} & =i \int d^{d} x e^{i q \cdot x}\left\langle 0\left|T\left(V_{\mu}^{i j}(x) S^{k l}(0)\right)\right| 0\right\rangle, \\
\Pi_{\mu}^{P}(q)^{i j k l} & =i \int d^{d} x e^{i q \cdot x}\left\langle 0\left|T\left(A_{\mu}^{i j}(x) P^{k l}(0)\right)\right| 0\right\rangle, \\
\Pi^{S}(q)^{i j k l} & =i \int d^{d} x e^{i q \cdot x}\left\langle 0\left|T\left(S^{i j}(x) S^{k l}(0)\right)\right| 0\right\rangle, \\
\Pi^{P}(q)^{i j k l} & =i \int d^{d} x e^{i q \cdot x}\left\langle 0\left|T\left(P^{i j}(x) P^{k l}(0)\right)\right| 0\right\rangle .
\end{aligned}
$$

The other possibilities vanish because of parity. The large $N_{c}$ limit requires these to be proportional to $\delta^{i l} \delta^{j k}$ and Lorentz and translational invariance allow them to be written in terms of functions that only depend on $q^{2}$ and the flavour index $i, j$.

$$
\Pi_{\mu \nu}^{V}(q)_{i j k l}=\left\{\left(q_{\mu} q_{\nu}-g_{\mu \nu} q^{2}\right) \Pi_{V i j}^{(1)}\left(q^{2}\right)+q_{\mu} q_{\nu} \Pi_{V i j}^{(0)}\left(q^{2}\right)\right\} \delta^{i l} \delta^{j k}
$$




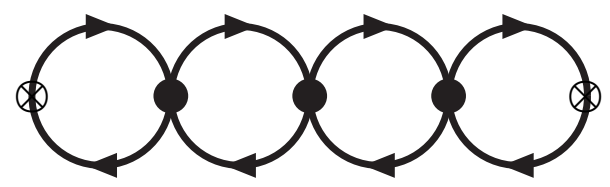

(a)

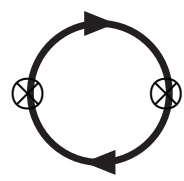

(b)

Figure 1: The type of diagrams in large $N_{c}$ that contribute to the two-point function. $\otimes$ indicates and insertion of an external current and $\bullet$ indicates the ENJL four-quark vertex. (a) the full two-point function. (b) The one-loop two-point function.

$$
\begin{aligned}
\Pi_{\mu \nu}^{A}(q)_{i j k l} & =\left\{\left(q_{\mu} q_{\nu}-g_{\mu \nu} q^{2}\right) \Pi_{A i j}^{(1)}\left(q^{2}\right)+q_{\mu} q_{\nu} \Pi_{A i j}^{(0)}\left(q^{2}\right)\right\} \delta^{i l} \delta^{j k} \\
\Pi_{\mu}^{S}(q)_{i j k l} & =q_{\mu} \Pi_{S i j}^{M}\left(q^{2}\right) \delta^{i l} \delta^{j k} \\
\Pi_{\mu}^{P}(q)_{i j k l} & =i q_{\mu} \Pi_{P i j}^{M}\left(q^{2}\right) \delta^{i l} \delta^{j k} \\
\Pi^{S}(q)_{i j k l} & =\Pi_{S i j}\left(q^{2}\right) \delta^{i l} \delta^{j k} \\
\Pi^{P}(q)_{i j k l} & =\Pi_{P i j}\left(q^{2}\right) \delta^{i l} \delta^{j k} .
\end{aligned}
$$

These functions satisfy Ward-identities following from chiral symmetry and the QCD equations of motion

$$
\begin{aligned}
q^{2} \Pi_{V i j}^{(0)}\left(q^{2}\right) & =\left(m_{i}-m_{j}\right) \Pi_{S i j}^{M}\left(q^{2}\right), \\
q^{2} \Pi_{S i j}^{M}\left(q^{2}\right) & =\left(m_{i}-m_{j}\right) \Pi_{S i j}\left(q^{2}\right)+\langle\bar{q} q\rangle_{i}-\langle\bar{q} q\rangle_{j}, \\
q^{2} \Pi_{A i j}^{(0)}\left(q^{2}\right) & =\left(m_{i}+m_{j}\right) \Pi_{P i j}^{M}\left(q^{2}\right), \\
q^{2} \Pi_{P i j}^{M}\left(q^{2}\right) & =\left(m_{i}+m_{j}\right) \Pi_{P i j}\left(q^{2}\right)+\langle\bar{q} q\rangle_{i}+\langle\bar{q} q\rangle_{j} .
\end{aligned}
$$

Here we use $\langle\bar{q} q\rangle_{i}=\sum_{\alpha}\left\langle 0\left|\bar{q}_{\alpha}^{i} q_{\alpha}^{i}\right| 0\right\rangle$.

The type of diagrams that contribute in large $N_{c}$ to the two-point functions is depicted in Fig. 1(a). The contribution from only the one-loop diagram is depicted in Fig. 1(b) and we will generally denote these as $\bar{\Pi}$.

Under interchange of $i$ and $j, \Pi_{S i j}^{M}\left(q^{2}\right)$ is anti-symmetric, all others are symmetric. The one-loop equivalents have the same symmetry properties.

In Refs. 7, 8] it was shown that the full two-point functions can be obtained from the one-loop ones via a resummation procedure

$$
\begin{aligned}
\Pi_{V i j}^{(1)}\left(q^{2}\right) & =\frac{\bar{\Pi}_{V i j}^{(1)}\left(q^{2}\right)}{1-q^{2} g_{V} \bar{\Pi}_{V i j}^{(1)}\left(q^{2}\right)} \\
\Pi_{V i j}^{(0)}\left(q^{2}\right) & =\frac{1}{\Delta_{S}\left(q^{2}\right)}\left[\left(1-g_{S} \bar{\Pi}_{S i j}\left(q^{2}\right)\right) \bar{\Pi}_{V i j}^{(0)}\left(q^{2}\right)+g_{S}\left(\bar{\Pi}_{S i j}^{M}\left(q^{2}\right)\right)^{2}\right] \\
\Pi_{S i j}^{M}\left(q^{2}\right) & =\frac{1}{\Delta_{S}\left(q^{2}\right)} \bar{\Pi}_{S i j}^{M}\left(q^{2}\right) \\
\Pi_{S i j}\left(q^{2}\right) & =\frac{1}{\Delta_{S}\left(q^{2}\right)}\left[\left(1+q^{2} g_{V} \bar{\Pi}_{V i j}^{(0)}\left(q^{2}\right)\right) \bar{\Pi}_{S i j}\left(q^{2}\right)-q^{2} g_{V}\left(\bar{\Pi}_{S i j}^{M}\left(q^{2}\right)\right)^{2}\right]
\end{aligned}
$$




$$
\begin{aligned}
\Delta_{S}\left(q^{2}\right) & =\left(1+q^{2} g_{V} \bar{\Pi}_{V i j}^{(0)}\left(q^{2}\right)\right)\left(1-g_{S} \bar{\Pi}_{S i j}\left(q^{2}\right)\right)+q^{2} g_{S} g_{V}\left(\bar{\Pi}_{S i j}^{M}\left(q^{2}\right)\right)^{2} \\
\Pi_{A i j}^{(1)}\left(q^{2}\right) & =\frac{\bar{\Pi}_{A i j}^{(1)}\left(q^{2}\right)}{1-q^{2} g_{V} \bar{\Pi}_{A i j}^{(1)}\left(q^{2}\right)} \\
\Pi_{A i j}^{(0)}\left(q^{2}\right) & =\frac{1}{\Delta_{P}\left(q^{2}\right)}\left[\left(1-g_{S} \bar{\Pi}_{P i j}\left(q^{2}\right)\right) \bar{\Pi}_{A i j}^{(0)}\left(q^{2}\right)+g_{S}\left(\bar{\Pi}_{P i j}^{M}\left(q^{2}\right)\right)^{2}\right] \\
\Pi_{P i j}^{M}\left(q^{2}\right) & =\frac{1}{\Delta_{P}\left(q^{2}\right)} \bar{\Pi}_{P i j}^{M}\left(q^{2}\right) \\
\Pi_{P i j}\left(q^{2}\right) & =\frac{1}{\Delta_{P}\left(q^{2}\right)}\left[\left(1+q^{2} g_{V} \bar{\Pi}_{A i j}^{(0)}\left(q^{2}\right)\right) \bar{\Pi}_{P i j}\left(q^{2}\right)-q^{2} g_{V}\left(\bar{\Pi}_{P i j}^{M}\left(q^{2}\right)\right)^{2}\right] \\
\Delta_{P}\left(q^{2}\right) & =\left(1+q^{2} g_{V} \bar{\Pi}_{A i j}^{(0)}\left(q^{2}\right)\right)\left(1-g_{S} \bar{\Pi}_{P i j}\left(q^{2}\right)\right)+q^{2} g_{S} g_{V}\left(\bar{\Pi}_{P i j}^{M}\left(q^{2}\right)\right)^{2}
\end{aligned}
$$

This resummation is only consistent with the Ward Identities, Eq. (5), if the one-loop two-point functions obey the Ward Identities of Eq. (515) with the current quark masses $m_{i}$ replaced by the constituent quark masses $M_{i}$ given by

$$
M_{i}=m_{i}-g_{S}\langle\bar{q} q\rangle_{i},
$$

known as the gap equation. The assumption of resummation thus leads to a constituent quark mass picture and one-loop Ward identities with constituent quark masses.

Using the gap equation and the one-loop Ward identities the resummation formulas can be simplified using

$$
\begin{aligned}
& \Delta_{S}\left(q^{2}\right)=1-g_{S} \bar{\Pi}_{S i j}\left(q^{2}\right)+g_{V}\left(m_{i}-m_{j}\right) \bar{\Pi}_{S i j}^{M}\left(q^{2}\right), \\
& \Delta_{P}\left(q^{2}\right)=1-g_{S} \bar{\Pi}_{P i j}\left(q^{2}\right)+g_{V}\left(m_{i}+m_{j}\right) \bar{\Pi}_{P i j}^{M}\left(q^{2}\right) .
\end{aligned}
$$

Our model assumption is to choose the one-loop functions as basic parameters rather than have them predicted via the constituent quark loops. This allows for a theory that has confinement built in a simple way and at the same time keeps most of the successes of the ENJL model in low-energy hadronic physics.

We now choose the two-point functions as far as possible as constants and have thus as parameters in the two-point sector

$$
\langle\bar{q} q\rangle_{i}, g_{S}, g_{V}, \bar{\Pi}_{P i j}^{M}, \bar{\Pi}_{A i j}^{(0+1)}, \bar{\Pi}_{S i j}^{M}, \bar{\Pi}_{V i j}^{(0+1)}
$$

and the remaining one-loop two-point functions can be obtained from the one-loop Ward identities. As discussed below, more input will be needed for the three-point functions. We do not expand higher in momenta in the one-loop two-point functions. The reason for this is that assuming that $g_{V}$ and $g_{S}$ are constants, expanding the one-loop two-point functions higher in momenta causes a gap in the large $q^{2}$ expansion between the leading and the non-leading terms. Such a gap in powers is not present as we know from perturbative QCD. 


\subsection{Chiral Limit}

In the chiral limit, the Ward identity for $\bar{\Pi}_{S i j}\left(q^{2}\right)$ becomes singular and it is better to choose instead as parameters

$$
\langle\bar{q} q\rangle_{\chi}, \Delta, g_{S}, g_{V}, \bar{\Pi}_{P}^{M \chi}, \bar{\Pi}_{A}^{(0+1) \chi}, \Gamma, \bar{\Pi}_{V}^{(0+1) \chi}
$$

with the parameters $\Delta, \Gamma$ defined via

$$
\begin{aligned}
\langle\bar{q} q\rangle_{i} & =\langle\bar{q} q\rangle_{\chi}+m_{i} \Delta+m_{i}^{2} \epsilon+\mathcal{O}\left(m_{i}^{3}\right), \\
\bar{\Pi}_{S i j}\left(q^{2}\right) & =q^{2} \Gamma-\frac{\Delta}{1-g_{S} \Delta}+\mathcal{O}\left(m_{i}, m_{j}\right) .
\end{aligned}
$$

\subsubsection{Short-Distance}

We define $\Pi_{L R}=\Pi_{V}-\Pi_{A}$ and $\Pi_{X}^{0+1}=\Pi_{X}^{(0)}+\Pi_{X}^{(1)}$ for $X=L R, V, A$ then the first and third Weinberg sum rules [15],

$$
\lim _{q^{2} \rightarrow-\infty}\left(q^{2} \Pi_{L R}^{(0+1) Q C D}\left(q^{2}\right)\right)=0 \text { and } \lim _{q^{2} \rightarrow-\infty}\left(q^{4} \Pi_{L R}^{(0) Q C D}\left(q^{2}\right)\right)=0,
$$

are automatically satisfied but the second one,

$$
\lim _{q^{2} \rightarrow-\infty}\left(q^{4} \Pi_{L R}^{(1) Q C D}\left(q^{2}\right)\right)=0
$$

implies the relation

$$
\bar{\Pi}_{A}^{(0+1) \chi}=\bar{\Pi}_{V}^{(0+1) \chi}
$$

Analogs of the Weinberg sum rules exist in scalar-pseudoscalar sector. With $\Pi_{S P}=$ $\Pi_{S}-\Pi_{P}$ we have [12, 16]

$$
\lim _{q^{2} \rightarrow-\infty} \prod_{S P i j}^{Q C D}\left(q^{2}\right)=0 \quad \text { and } \quad \lim _{q^{2} \rightarrow-\infty}\left(q^{2} \prod_{S P i j}^{Q C D}\left(q^{2}\right)\right)=0
$$

The first one is the equivalent of the first Weinberg sum rule and is automatically satisfied. The second one implies

$$
\Gamma=\frac{-\bar{\Pi}_{P}^{M \chi}}{2 g_{S}\langle\bar{q} q\rangle_{\chi}\left(1-2 g_{S} g_{V}\langle\bar{q} q\rangle_{\chi} \bar{\Pi}_{P}^{M \chi}\right)} .
$$

The short-distance relation found in Eq. (17) does not satisfy the heat kernel relation for the one-loop two-point functions derived in [7] in the chiral limit. Note that that heat

kernel relation was the underlying cause of the relation $m_{S}=2 M_{q}$ between the scalar mass and the constituent quark mass in ENJL models [7, 8]. 


\subsubsection{Intermediate-Distance}

The two-point functions in the chiral limit can be written as

$$
\begin{aligned}
\Pi_{V}^{(1) \chi}\left(q^{2}\right) & =\frac{2 f_{V}^{2} m_{V}^{2}}{m_{V}^{2}-q^{2}}, \\
\Pi_{A}^{(1) \chi}\left(q^{2}\right) & =\frac{-2 F_{0}^{2}}{q^{2}}+\frac{2 f_{A}^{2} m_{A}^{2}}{m_{A}^{2}-q^{2}}, \\
\Pi_{P}^{M \chi}\left(q^{2}\right) & =\frac{2\langle\bar{q} q\rangle_{\chi}}{q^{2}}, \\
\Pi_{S}^{\chi}\left(q^{2}\right) & =K_{S}+\frac{2 F_{S}^{2} m_{S}^{2}}{m_{S}^{2}-q^{2}} \\
\Pi_{P}^{\chi}\left(q^{2}\right) & =K_{P}-\frac{2 F_{0}^{2} B_{0}^{2}}{q^{2}}
\end{aligned}
$$

From the poles in the two-point functions we can find the various masses. There is a pole at $q^{2}=0$ corresponding to the massless pion. The scalar, vector and axial-vector masses are given by

$$
\begin{aligned}
m_{S}^{2} & =\frac{1}{g_{S} \Gamma\left(1-g_{S} \Delta\right)}, \\
m_{V}^{2} & =\frac{1}{g_{V} \bar{\Pi}_{V}^{(0+1) \chi}}, \\
m_{A}^{2} & =\frac{1-2 g_{S} g_{V}\langle\bar{q} q\rangle_{\chi} \bar{\Pi}_{P}^{M \chi}}{g_{V} \bar{\Pi}_{A}^{(0+1) \chi}}=\left(1-2 g_{S} g_{V}\langle\bar{q} q\rangle_{\chi} \bar{\Pi}_{P}^{M \chi}\right) m_{V}^{2} .
\end{aligned}
$$

The residues at the poles lead to

$$
\begin{aligned}
2 f_{V}^{2} & =\bar{\Pi}_{V}^{(0+1) \chi}, \\
2 f_{A}^{2} & =\frac{\bar{\Pi}_{V}^{(0+1) \chi}}{\left(1-2 g_{S} g_{V}\langle\bar{q} q\rangle_{\chi} \bar{\Pi}_{P}^{M \chi}\right)^{2}}, \\
2 F_{0}^{2} & =\frac{-2 g_{S}\langle\bar{q} q\rangle_{\chi} \bar{\Pi}_{P}^{M \chi}}{1-2 g_{S} g_{V}\langle\bar{q} q\rangle_{\chi} \bar{\Pi}_{P}^{M \chi}}, \\
K_{S} & =K_{P}=-\frac{1}{g_{S}} \\
2 F_{S}^{2} & =\frac{1-g_{S} \Delta}{g_{S}}, \\
B_{0}^{2} F_{0}^{4} & =\langle\bar{q} q\rangle_{\chi}^{2}
\end{aligned}
$$

The short distance constraints lead as expected to

$$
f_{V}^{2} m_{V}^{2}=f_{A}^{2} m_{A}^{2}+F_{0}^{2},
$$




$$
\begin{aligned}
f_{V}^{2} m_{V}^{4} & =f_{A}^{2} m_{A}^{4} \\
K_{S} & =K_{P} \\
F_{S}^{2} m_{S}^{2} & =F_{0}^{2} B_{0}^{2}
\end{aligned}
$$

\subsubsection{Long-Distance}

The two-point functions in the chiral limit can be determined from Chiral Perturbation Theory. This lead to the identification of $B_{0}, F_{0}$ with the quantities appearing there and in addition

$$
\begin{aligned}
L_{10} & =-\frac{1}{4}\left(f_{V}^{2}-f_{A}^{2}\right), & H_{1}=-\frac{1}{8}\left(f_{V}^{2}+f_{A}^{2}\right), \\
32 B_{0}^{2} L_{8} & =2 F_{S}^{2}, & 16 B_{0}^{2} H_{2}=2 K_{S}+2 F_{S}^{2}
\end{aligned}
$$

\subsubsection{Parameters}

Notice that from the six input parameters we can only determine five from the two-point function inputs. A possible choice of input parameters is $m_{V}, m_{A}, F_{0}, m_{S}$ and $F_{S}$. The last can be traded for $B_{0}$ or $\langle\bar{q} q\rangle_{\chi}$. The remaining parameter could in principle be fixed from $K_{S}$ but that is an unmeasurable quantity.

\subsection{Beyond the Chiral Limit}

The resummation formulas of Sect. 2.1 remain valid. What changes now is that we have values for the current quark masses $m_{i}$ and corresponding changes in the one-loop functions. An underlying expectation is that the vertices $g_{S}$ and $g_{V}$ are produced by purely gluonic effects and have no light quark-mass dependence. The first order the quark-mass dependence of $g_{V}$ and $g_{S}$ must be zero from short-distance constraints as shown below.

The input parameters are now given by Eq. (10) and we will below expand them as functions in $m_{q}$.

\subsubsection{Intermediate-Distance}

The resummation leads to expressions for the two-point functions which can again be written as one resonance exchange.

$$
\begin{aligned}
\Pi_{V i j}^{(1)}\left(q^{2}\right) & =-\frac{2 f_{S i j}^{2}}{q^{2}}+\frac{2 f_{V i j}^{2} m_{V i j}^{2}}{m_{V i j}^{2}-q^{2}}, \\
\Pi_{V i j}^{(0)}\left(q^{2}\right) & =2 f_{S i j}^{2}\left(\frac{1}{m_{S i j}^{2}-q^{2}}+\frac{1}{q^{2}}\right), \\
\Pi_{A i j}^{(1)}\left(q^{2}\right) & =-\frac{2 f_{i j}^{2}}{q^{2}}+\frac{2 f_{A i j}^{2} m_{A i j}^{2}}{m_{A i j}^{2}-q^{2}},
\end{aligned}
$$




$$
\begin{aligned}
\Pi_{A i j}^{(0)}\left(q^{2}\right) & =2 f_{i j}^{2}\left(\frac{1}{m_{i j}^{2}-q^{2}}+\frac{1}{q^{2}}\right), \\
\Pi_{S i j}^{M}\left(q^{2}\right) & =\frac{2 F_{S i j} m_{S i j} f_{S i j}}{m_{S i j}^{2}-q^{2}}, \\
\Pi_{P i j}^{M}\left(q^{2}\right) & =\frac{2 B_{i j} f_{i j}^{2}}{m_{i j}^{2}-q^{2}}, \\
\Pi_{S i j}\left(q^{2}\right) & =K_{S i j}+\frac{2 F_{S i j}^{2} m_{S i j}^{2}}{m_{S i j}^{2}-q^{2}}, \\
\Pi_{P i j}\left(q^{2}\right) & =K_{P i j}+\frac{2 f_{i j}^{2} B_{i j}^{2}}{m_{i j}^{2}-q^{2}} .
\end{aligned}
$$

These satisfy the Ward Identities (5). The values of the couplings and masses are given by

$$
\begin{aligned}
& m_{V i j}^{2}=\frac{1+g_{V} \bar{\Pi}_{S i j}^{M}\left(M_{i}-M_{j}\right)}{g_{V} \bar{\Pi}_{V i j}^{(0+1)}}, \\
& m_{A i j}^{2}=\frac{1+g_{V} \bar{\Pi}_{P i j}^{M}\left(M_{i}+M_{j}\right)}{g_{V} \bar{\Pi}_{A i j}^{(0+1)}}, \\
& m_{S i j}^{2}=\frac{m_{i}-m_{j}}{\bar{\Pi}_{S i j}^{M}} \frac{\left(1+g_{V} \bar{\Pi}_{S i j}^{M}\left(M_{i}-M_{j}\right)\right)}{g_{S}}, \\
& m_{i j}^{2}=\left(m_{i}+m_{j}\right) \frac{1+g_{V}\left(M_{i}+M_{j}\right) \bar{\Pi}_{P i j}^{M}}{g_{S} \bar{\Pi}_{P i j}^{M}}, \\
& 2 f_{V i j}^{2}=\frac{\bar{\Pi}_{V i j}^{(0+1)}}{\left(1+g_{V} \bar{\Pi}_{S i j}^{M}\left(M_{i}-M_{j}\right)\right)^{2}}, \\
& 2 f_{A i j}^{2}=\frac{\bar{\Pi}_{A i j}^{(0+1)}}{\left(1+g_{V} \bar{\Pi}_{P i j}^{M}\left(M_{i}+M_{j}\right)\right)^{2}}, \\
& 2 F_{S i j}^{2}=\frac{M_{i}-M_{j}}{g_{S}\left(m_{i}-m_{j}\right)} \text {, } \\
& 2 f_{S i j}^{2}=\frac{\left(M_{i}-M_{j}\right) \bar{\Pi}_{S i j}^{M}}{1+g_{V} \bar{\Pi}_{S i j}^{M}\left(M_{i}-M_{j}\right)}, \\
& 2 f_{i j}^{2}=\frac{\left(M_{i}+M_{j}\right) \bar{\Pi}_{P i j}^{M}}{1+g_{V} \bar{\Pi}_{P i j}^{M}\left(M_{i}+M_{j}\right)}, \\
& K_{S i j}=K_{P i j}=-\frac{1}{g_{S}} \text {, } \\
& B_{i j}=\frac{1+g_{V}\left(M_{i}+M_{j}\right) \bar{\Pi}_{P i j}^{M}}{g_{S} \bar{\Pi}_{P i j}^{M}} .
\end{aligned}
$$




\subsubsection{Short-Distance}

In order to proceed we have to expand the input parameters of Eq. (10) in the quark masses $m_{q}$.

$$
\begin{aligned}
\bar{\Pi}_{V i j}^{(0+1)} & =\bar{\Pi}_{V}^{(0+1) \chi}+\left(m_{i}+m_{j}\right) \bar{\Pi}_{V}^{(0+1) I}+\mathcal{O}\left(m_{q}^{2}\right) \\
\bar{\Pi}_{A i j}^{(0+1)} & =\bar{\Pi}_{A}^{(0+1) \chi}+\left(m_{i}+m_{j}\right) \bar{\Pi}_{A}^{(0+1) I}+\mathcal{O}\left(m_{q}^{2}\right) \\
\bar{\Pi}_{P i j}^{M} & =\bar{\Pi}_{P}^{M \chi}+\left(m_{i}+m_{j}\right) \bar{\Pi}_{P}^{M I}+\mathcal{O}\left(m_{q}^{2}\right) \\
\bar{\Pi}_{S i j}\left(q^{2}\right) & =q^{2}\left(\Gamma+\left(m_{i}+m_{j}\right) \Gamma^{I}\right)-\frac{\Delta}{1-g_{S} \Delta}-\frac{\epsilon}{\left(1-g_{S} \Delta\right)^{2}}\left(m_{i}+m_{j}\right)+\mathcal{O}\left(m_{q}^{2}\right),
\end{aligned}
$$

The parameters $\epsilon$ and $\Delta$ are defined in the first line of Eq. (121). The other one-loop two-point functions are derivable from the one-loop Ward identities.

The chiral limit short-distance constraints Eqs. (15) and (17) remain valid but there are new constraints on the coefficients of the quark mass expansions. The derivatives w.r.t. the quark masses of the two-point functions allow to construct more order parameters than $\Pi_{L R}$ and $\Pi_{S P}$. In particular we have ${ }^{2}$

$$
\begin{aligned}
\lim _{q^{2} \rightarrow-\infty} \lim _{m_{q} \rightarrow 0}\left(q^{4} \frac{\partial}{\partial m_{i}} \Pi_{V i j}^{(1)}\left(q^{2}\right)\right) & =\langle\bar{q} q\rangle_{\chi}, \\
\lim _{q^{2} \rightarrow-\infty} \lim _{m_{q} \rightarrow 0}\left(q^{4} \frac{\partial}{\partial m_{i}} \Pi_{V i j}^{(0)}\left(q^{2}\right)\right) & =0, \\
\lim _{q^{2} \rightarrow-\infty} \lim _{m_{q} \rightarrow 0}\left(q^{4} \frac{\partial}{\partial m_{i}} \Pi_{A i j}^{(1)}\left(q^{2}\right)\right) & =-\langle\bar{q} q\rangle_{\chi}, \\
\lim _{q^{2} \rightarrow-\infty} \lim _{m_{q} \rightarrow 0}\left(q^{4} \frac{\partial}{\partial m_{i}} \Pi_{A i j}^{(0)}\left(q^{2}\right)\right) & =2\langle\bar{q} q\rangle_{\chi}, \\
\lim _{q^{2} \rightarrow-\infty} \lim _{m_{q} \rightarrow 0}\left(q^{2} \frac{\partial}{\partial m_{i}} \Pi_{S i j}\left(q^{2}\right)\right) & =-\frac{3}{2}\langle\bar{q} q\rangle_{\chi}, \\
\lim _{q^{2} \rightarrow-\infty} \lim _{m_{q} \rightarrow 0}\left(q^{2} \frac{\partial}{\partial m_{i}} \Pi_{P i j}\left(q^{2}\right)\right) & =\frac{1}{2}\langle\bar{q} q\rangle_{\chi} .
\end{aligned}
$$

The ones with lower powers of $q^{2}$ must vanish. The second and fourth are automatically satisfied as a consequence from the Ward identities. $\Pi_{V i j}^{(0)}\left(q^{2}\right)$ only starts at $\mathcal{O}\left(m_{q}^{2}\right)$ and the $m_{i}+m_{j}$ term in $\Pi_{A i j}^{(0)}\left(q^{2}\right)$ follows from the Ward identity and the chiral limit form of $\Pi_{P i j}^{M}$.

The vanishing of those with lower powers of $q^{2}$ requires that

$$
\lim _{m_{q} \rightarrow 0} \frac{\partial g_{V}}{\partial m_{i}}=\lim _{m_{q} \rightarrow 0} \frac{\partial g_{S}}{\partial m_{i}}=0 .
$$

\footnotetext{
${ }^{2}$ We have derived these expressions but they can also be found in [16].
} 
The first, third, fifth and sixth identities give

$$
\begin{aligned}
\bar{\Pi}_{V}^{(0+1) I} & =g_{V}^{2}\left(\bar{\Pi}_{V}^{(0+1) \chi}\right)^{2}\langle\bar{q} q\rangle_{\chi}, \\
\bar{\Pi}_{A}^{(0+1) I} & =-g_{V}^{2}\left(\bar{\Pi}_{V}^{(0+1) \chi}\right)^{2}\langle\bar{q} q\rangle_{\chi}, \\
\Gamma^{I} & =-\frac{3}{2} g_{S}^{2} \Gamma^{2}\langle\bar{q} q\rangle_{\chi}, \\
\bar{\Pi}_{P}^{M I} & =-\frac{1}{4} g_{S}\left(\bar{\Pi}_{P}^{M \chi}\right)^{2}-\frac{1-g_{S} \Delta}{2 g_{S}\langle\bar{q} q\rangle_{\chi}} \bar{\Pi}_{P}^{M \chi}\left(1-4 g_{V} g_{S}\langle\bar{q} q\rangle_{\chi} \bar{\Pi}_{P}^{M \chi}\right) .
\end{aligned}
$$

This implies that the only new parameter that appears to include quark masses to first order is $\epsilon$. The last constraint turns out to be incompatible with short-distance constraints from three-point functions as discussed below.

\subsubsection{Long-Distance}

The long-distance expansion of our results to $\mathcal{O}\left(p^{4}\right)$ in Chiral Perturbation Theory allows in addition to those already obtained in the chiral limit also

$$
L_{5}=\frac{1}{16} F_{0}^{6}\left[\frac{\bar{\Pi}_{P}^{M \chi}\left(g_{S} \Delta-1\right)+2 g_{S}\langle\bar{q} q\rangle_{\chi} \bar{\Pi}_{P}^{M I}}{\left(\bar{\Pi}_{P}^{M \chi}\right)^{2} g_{S}^{2}\langle\bar{q} q\rangle_{\chi}^{3}}\right] .
$$

\subsubsection{Intermediate-Distance}

The short-distance constraints lead to several relations between resonance parameters also beyond the chiral limit to first order in current quark masses. In the vector sector we obtain

$$
\begin{aligned}
f_{V i j}^{2} m_{V i j}^{2} & =f_{V k l}^{2} m_{V k l}^{2}, \\
f_{V i j}^{2} m_{V i j}^{4}-f_{V k l}^{2} m_{V k l}^{4} & =-\frac{1}{2}\langle\bar{q} q\rangle_{\chi}\left(m_{i}+m_{j}-m_{k}-m_{l}\right) .
\end{aligned}
$$

$V_{i j}$ stands here for the vector degree of freedom built of quarks with current mass $m_{i}$ and $m_{j}$.

The corresponding axial relations are

$$
\begin{aligned}
f_{A i j}^{2} m_{A i j}^{2}+f_{i j}^{2} & =f_{A k l}^{2} m_{A k l}^{2}+f_{k l}^{2}, \\
f_{A i j}^{2} m_{A i j}^{4}-f_{A k l}^{2} m_{A k l}^{4} & =\frac{1}{2}\langle\bar{q} q\rangle_{\chi}\left(m_{i}+m_{j}-m_{k}-m_{l}\right) .
\end{aligned}
$$

\section{Three-Point Functions}

A generic three-point function of currents $A, B, C$ chosen from the currents in Eq. (2) is defined as

$$
\Pi^{A B C}\left(p_{1}, p_{2}\right)^{i j k l m n}=i^{2} \int d^{d} x d^{d} y e^{i p_{1} \cdot x} e^{i p_{2} \cdot y}\left\langle 0\left|T\left(A^{i j}(0) B^{k l}(x) C^{m n}(y)\right)\right| 0\right\rangle .
$$




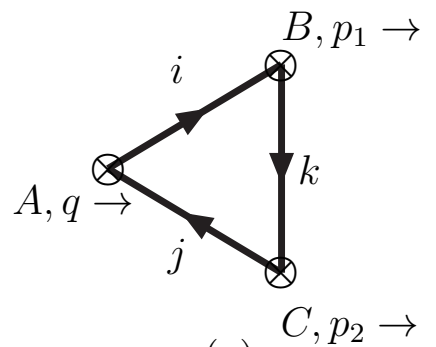

(a)

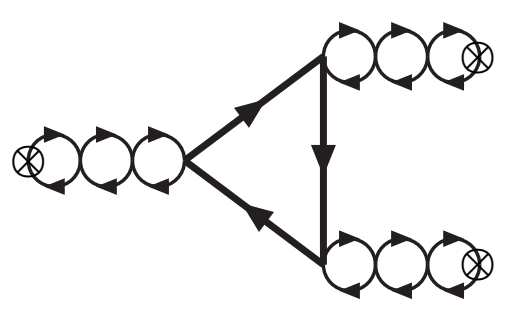

(b)

Figure 2: The $\Pi^{+}$contribution to a generic three-point function. (a) The flavour and momentum flow indicated on a one-loop diagram. (b) A generic large $N_{c}$ diagram with the resummation in terms of bubbles. Note that the resummation leads to full two-point functions.

In the large $N_{c}$ limit these can only have two types of flavour flow

$$
\Pi^{A B C}\left(p_{1}, p_{2}\right)^{i j k l m n}=\Pi^{A B C+}\left(p_{1}, p_{2}\right)^{i j k} \delta^{i l} \delta^{j m} \delta^{k n}+\Pi^{A B C-}\left(p_{1}, p_{2}\right)^{i j l} \delta^{i n} \delta^{j k} \delta^{l m}
$$

and they satisfy

$$
\Pi^{A B C-}\left(p_{1}, p_{2}\right)^{i j l}=\Pi^{A C B+}\left(p_{2}, p_{1}\right)^{i j l} .
$$

The flavour and momentum flow of $\Pi^{A B C+}\left(p_{1}, p_{2}\right)^{i j k}$ is indicated in Fig. 2(a). In the remainder we will always talk about the $\Pi^{+}$part only but drop the superscript + . We also use $q=p_{1}+p_{2}$. A generic contribution to the three-point function is shown in Fig. 2(b). The internal vertices are given by $g_{V}$ and $g_{S}$. In Ref. [8] it was shown on two examples how this resummation can be performed for some three-point functions. Many other cases were worked out for the work on non-leptonic matrix-elements in Refs. 13, 14].

Here we will make the assumption of resummation for the three-point functions just as we did for the two-point functions in Sect. 2. It can again be shown that the Ward identities for the full three-point functions and the resummation together require that the one-loop three-point functions satisfy the one-loop Ward identities with the constituent masses given by the gap equation (8).

We will once more assume that the three-point functions are constants or low-order polynomials of the kinematical variables, in agreement with the large $N_{c}$ limit Green functions structure. It turns out that the combination of one-loop Ward identities and short distance constraints is very powerful in restricting the number of new free parameters appearing in the three-point functions. This could already be seen in Sect. 2.3. since the derivative w.r.t. a quark mass of a two-point function is a three-point function with one of the momenta equal to zero.

A full analysis of three-point functions is in progress. Here we give a few representative examples. 


\subsection{The Pseudoscalar-Scalar-Pseudoscalar Three-Point Function and the Scalar Form Factor}

The Pseudoscalar-Scalar-Pseudoscalar three-point function can be calculated from the class of diagrams depicted in Fig. 2(b) using the methods of [8] and reads for the case of $m_{i}=m_{k}$

$$
\begin{aligned}
\Pi^{P S P}\left(p_{1}, p_{2}\right)^{i j k} \equiv & \left\{1+g_{S} \Pi_{S}\left(p_{1}^{2}\right)_{k i}\right\} \\
& \times\left\{\bar{\Pi}^{P S P}\left(p_{1}, p_{2}\right)^{i j k}\left(1+g_{S} \Pi_{P}\left(q^{2}\right)_{j i}\right)\left(1+g_{S} \Pi_{P}\left(p_{2}^{2}\right)_{j k}\right)\right. \\
& +\bar{\Pi}_{\mu}^{A S P}\left(p_{1}, p_{2}\right)^{i j k}\left(-g_{V} i q^{\mu} \Pi_{P}^{M}\left(q^{2}\right)_{j i}\right)\left(1+g_{S} \Pi_{P}\left(p_{2}^{2}\right)_{j k}\right) \\
& +\bar{\Pi}_{\nu}^{P S A}\left(p_{1}, p_{2}\right)^{i j k}\left(1+g_{S} \Pi_{P}\left(q^{2}\right)_{j i}\right)\left(g_{V} i p_{2}^{\nu} \Pi_{P}^{M}\left(p_{2}^{2}\right)_{j k}\right) \\
& \left.+\bar{\Pi}_{\mu \nu}^{A S A}\left(p_{1}, p_{2}\right)^{i j k}\left(-g_{V} i q^{\mu} \Pi_{P}^{M}\left(q^{2}\right)_{j i}\right)\left(g_{V} i p_{2}^{\nu} \Pi_{P}^{M}\left(p_{2}^{2}\right)_{j k}\right)\right\} .
\end{aligned}
$$

The general case has also terms involving one-loop three-point functions with a vector $(V)$ instead of a scalar $(S)$. The one-loop Ward identities can be used to rewrite $\bar{\Pi}^{A S P}, \bar{\Pi}^{P S A}$ and $\bar{\Pi}^{A S A}$ in terms of $\bar{\Pi}^{P S P}$ and one-loop two-point functions.

The one-loop three-point function $\bar{\Pi}^{P S P}$ is in turn fully fixed by the one-loop Ward Identities. Let us illustrate the derivation, one Ward Identity is

$$
i p_{2}^{\mu} \bar{\Pi}_{\mu}^{P S A}\left(p_{1}, p_{2}\right)^{i j k}=-\left(M_{j}+M_{k}\right) \bar{\Pi}^{P S P}\left(p_{1}, p_{2}\right)^{i j k}+\bar{\Pi}_{S i k}\left(p_{1}^{2}\right)-\bar{\Pi}_{P i j}\left(q^{2}\right) .
$$

Putting $p_{1}^{2}=p_{2}^{2}=q^{2}=0$ this determines

$$
\bar{\Pi}^{P S P}(0,0)^{i j k}=\frac{1}{M_{j}+M_{k}}\left\{\frac{\langle\bar{q} q\rangle_{k}-\langle\bar{q} q\rangle_{i}}{M_{i}-M_{k}}+\frac{\langle\bar{q} q\rangle_{i}+\langle\bar{q} q\rangle_{j}}{M_{i}+M_{j}}\right\} .
$$

The same result follows from the identities for $q^{\mu} \bar{\Pi}_{\mu}^{A S P}\left(p_{1}, p_{2}\right)^{i j k}$ and $p_{1}^{\mu} \bar{\Pi}_{\mu}^{P V P}\left(p_{1}, p_{2}\right)^{i j k}$.

The next term, linear in $q^{2}, p_{1}^{2}, p_{2}^{2}$, can be derived as well, since the relevant combinations of the three-point functions with one vector or axial-vector can be determined from Ward identities involving three-point functions with two vector or axial-vector currents.

We only quote here the chiral limit result

$$
\begin{aligned}
\bar{\Pi}^{P S P}\left(p_{1}, p_{2}\right)^{\chi}= & \frac{1}{2 g_{S}^{2}\left(1-g_{S} \Delta\right)\langle\bar{q} q\rangle_{\chi}}-\frac{p_{1}^{2}}{8 g_{S}\langle\bar{q} q\rangle_{\chi}}\left(4 \Gamma-\frac{2 \bar{\Pi}_{P}^{M I}}{\left(1-g_{S} \Delta\right)}+\frac{\bar{\Pi}_{P}^{M \chi}}{g_{S}\langle\bar{q} q\rangle_{\chi}}\right) \\
& -\frac{q^{2}+p_{2}^{2}}{8 g_{S}\langle\bar{q} q\rangle_{\chi}}\left(\frac{2 \bar{\Pi}_{P}^{M I}}{\left(1-g_{S} \Delta\right)}+\frac{\bar{\Pi}_{P}^{M \chi}}{g_{S}\langle\bar{q} q\rangle_{\chi}}\right) .
\end{aligned}
$$

From the $q^{2}$ dependence of the full Green-function at low energies we can also derive $L_{5}$, the result agrees with Eq. (29) as it should.

We can look at two different types of short-distance constraints. First, using the methods of exclusive processes in perturbative QCD [17, it can be shown that the scalar form factor in the chiral limit should decrease as $1 / p_{1}^{2}$. Phenomenologically, this short-distance 
behaviour has been also imposed in [18] to calculate the scalar form factor. It was checked that this behaviour agrees with data. Using the LSZ reduction formulas the scalar form factor of the pion in the chiral limit is

$$
F_{S}^{\chi}\left(p_{1}^{2}\right)=\lim _{q^{2}, p_{2}^{2} \rightarrow 0} \frac{q^{2} p_{2}^{2}}{-2 F_{0}^{2} B_{0}^{2}} \Pi^{P S P}\left(p_{1}, p_{2}\right)^{\chi}
$$

and it can be written in a simpler form ${ }^{3}$

$$
F_{S}^{\chi}\left(p_{1}^{2}\right)=B_{0} \frac{m_{S}^{2}}{m_{S}^{2}-p_{1}^{2}}\left(1+p_{1}^{2}\left(\frac{4 L_{5}}{F_{0}^{2}}-\frac{1}{m_{S}^{2}}\right)\right) .
$$

The short-distance requirement on $F_{S}^{\chi}\left(p_{1}^{2}\right)$ thus requires $L_{5}$ to have its resonance dominated value

$$
L_{5}=\frac{F_{0}^{2}}{4 m_{S}^{2}} .
$$

This gives a new relation between the input parameters, after using Eq. (17),

$$
\bar{\Pi}_{P}^{M I}=\frac{\left(1-g_{S} \Delta\right) \bar{\Pi}_{P}^{M \chi}}{2 g_{S}\langle\bar{q} q\rangle_{\chi}}\left(-1+4 g_{V} g_{S}\langle\bar{q} q\rangle_{\chi} \bar{\Pi}_{P}^{M \chi}\right) .
$$

This constraint is not compatible with Eq. (28).

The three-point function $\Pi^{P S P}\left(p_{1}, p_{2}\right)^{i j k}$ is an order parameter in the sense described above. Its short-distance properties can thus be used to constrain the theory. The shortdistance behaviour is

$$
\lim _{\lambda \rightarrow \infty} \Pi^{P S P}\left(\lambda p_{1}, \lambda p_{2}\right)^{\chi}=0 .
$$

This is automatically satisfied by our expression (44).

The entire $\Pi^{P S P \chi}$ can be written in a simple fashion

$$
\Pi^{P S P}\left(p_{1}, p_{2}\right)^{\chi}=-2 F_{0}^{2} B_{0}^{3} \frac{m_{S}^{2}}{q^{2} p_{2}^{2}\left(m_{S}^{2}-p_{1}^{2}\right)}\left(1+b\left(q^{2}+p_{2}^{2}-p_{1}^{2}\right)\right)
$$

with

$$
b=0(\text { Eq. (42) }) \quad \text { or } \quad b=\frac{F_{0}^{4}}{8\langle\bar{q} q\rangle_{\chi}^{2}}=\frac{1}{8 B_{0}^{2}}(\text { Eq. (28) }) .
$$

The short distance relation $\lim _{\lambda \rightarrow \infty} F_{S}^{\chi}\left(\lambda p_{1}^{2}\right)=0$ has no $\alpha_{S}$ corrections. We therefore consider the constraint Eq. (42) to be more reliable than the one from Eq. (28).

\footnotetext{
${ }^{3}$ Notice that in order to have the usual scalar form factor we need to add the $\Pi^{+}$and $\Pi^{-}$of Eq. (33). The formulas here refer only to $\Pi^{+}$.
} 


\subsection{The Vector-Pseudoscalar-Pseudoscalar Three-Point Function and the Vector Form Factor}

We can repeat the analysis of Sect. 3.1 now for the VPP three-point function. The results will be very similar to there and apply to the vector (electromagnetic) form factor. We keep here to the simpler case of $m_{i}=m_{j}$. The resummation leads to [8]

$$
\begin{aligned}
\Pi_{\mu}^{V P P}\left(p_{1}, p_{2}\right)^{i j k}= & \left\{g^{\mu \nu}-g_{V} \Pi_{\mu \nu}^{V}(q)^{i j}\right\} \\
& \times\left\{\bar{\Pi}_{\nu}^{V P P}\left(p_{1}, p_{2}\right)^{i j k}\left(1+g_{S} \Pi_{P i k}\left(p_{1}^{2}\right)\right)\left(1+g_{S} \Pi_{P k j}\left(p_{2}^{2}\right)\right)\right. \\
& +\bar{\Pi}_{\nu \beta}^{V P A}\left(p_{1}, p_{2}\right)^{i j k}\left(1+g_{S} \Pi_{P i k}\left(p_{1}^{2}\right)\right)\left(g_{V} i p_{2}^{\beta} \Pi_{P k j}^{M}\left(p_{2}^{2}\right)\right) \\
& +\bar{\Pi}_{\nu \alpha}^{V A P}\left(p_{1}, p_{2}\right)^{i j k}\left(g_{V} i p_{1}^{\alpha} \Pi_{P i k}^{M}\left(p_{1}^{2}\right)\right)\left(1+g_{S} \Pi_{P k j}\left(p_{2}^{2}\right)\right) \\
& \left.+\bar{\Pi}_{\nu \alpha \beta}^{V A A}\left(p_{1}, p_{2}\right)^{i j k}\left(g_{V} i p_{1}^{\alpha} \Pi_{P i k}^{M}\left(p_{1}^{2}\right)\right)\left(g_{V} i p_{2}^{\beta} \Pi_{P k j}^{M}\left(p_{2}^{2}\right)\right)\right\} .
\end{aligned}
$$

We can again use the Ward Identities to rewrite this in terms of two-point functions and $\bar{\Pi}_{\mu}^{V P P}\left(p_{1}, p_{2}\right)^{i j k}$ only.

We now expand in $p_{1}^{2}, p_{2}^{2}$ and $\left(p_{1}+p_{2}\right)^{2}=q^{2}$.

$$
\bar{\Pi}_{\mu}^{V P P}\left(p_{1}, p_{2}\right)^{i j k}=p_{1 \mu} \bar{\Pi}_{1}^{V P P i j k}+p_{2 \mu} \bar{\Pi}_{2}^{V P P i j k}+C_{i j k}^{V P P}\left(q \cdot p_{2} p_{1 \mu}-q \cdot p_{1} p_{2 \mu}\right) .
$$

The one-loop WI imply

$$
\begin{aligned}
& \bar{\Pi}_{1}^{V P P i j k}=\frac{-\bar{\Pi}_{S i j}^{M}+\bar{\Pi}_{P i k}^{M}}{M_{j}+M_{k}} \\
& \bar{\Pi}_{2}^{V P P i j k}=\frac{-\bar{\Pi}_{S i j}^{M}-\bar{\Pi}_{P j k}^{M}}{M_{i}+M_{k}} .
\end{aligned}
$$

The next term in the expansion depends only on one constant. This follows from the assumption (in the previous subsection) that $\bar{\Pi}^{S P P}$ contains no terms more than linear in $p_{1}^{2}, p_{2}^{2}, q^{2}$. The form given in Eq. (47) includes this assumption already. This extra constant can be determined from the fact that the pion vector factor should decrease as $1 / q^{2}$ for large $q^{2}$. Extracting the chiral limit ${ }^{4}$ vector form factor via

$$
F_{V}^{\chi}\left(q^{2}\right)=\lim _{p_{1}^{2}, p_{2}^{2} \rightarrow 0} \frac{p_{1}^{2} p_{2}^{2}}{2 F_{0}^{2} B_{0}^{2}} \Pi_{1}^{V P P}\left(p_{1}, p_{2}\right)^{\chi} .
$$

The subscript one means the coefficient of $p_{1 \mu}$ in the expansion

$$
\Pi_{\mu}^{V P P}\left(p_{1}, p_{2}\right)=p_{1 \mu} \Pi_{1}^{V P P}\left(p_{1}, p_{2}\right)+p_{2 \mu} \Pi_{2}^{V P P}\left(p_{1}, p_{2}\right) .
$$

\footnotetext{
${ }^{4}$ This argument is also valid outside the chiral limit.
} 
The short-distance requirement then determines

$$
C_{\chi}^{V P P}=\left(\bar{\Pi}_{P}^{M \chi}\right)^{2} g_{V}^{2} \bar{\Pi}_{V}^{(0+1) \chi} .
$$

The ChPT expression for the pion vector form factor yields then

$$
L_{9}=\frac{F_{0}^{2}}{2} g_{V} \bar{\Pi}_{V}^{(0+1) \chi}=\frac{1}{2} \frac{F_{0}^{2}}{m_{V}^{2}} .
$$

The full chiral limit three-point function can be written in a simple fashion

$$
\Pi_{\mu}^{V P P}\left(p_{1}, p_{2}\right)^{\chi}=\frac{-2 F_{0}^{2} B_{0}^{2}}{p_{1}^{2} p_{2}^{2}} \frac{m_{V}^{2}}{m_{V}^{2}-q^{2}}\left(p_{1 \mu}-p_{2 \mu}+A\left(p_{2}^{2}-p_{1}^{2}\right)\left(p_{1 \mu}+p_{2 \mu}\right)\right),
$$

with

$$
A=g_{V} \bar{\Pi}_{V}^{(0+1) \chi}=\frac{1}{m_{V}^{2}} .
$$

\subsection{The Scalar-Vector-Vector Three-Point function}

The Scalar-Vector-Vector three-point function has been used to discuss the properties of

the scalars in Ref. [12. The relation between the full and the one-loop functions in the case of all masses equal is

$$
\begin{aligned}
\Pi_{\mu \nu}^{S V V}\left(p_{1}, p_{2}\right)^{i i i} \equiv & \left\{g_{\mu \alpha}-g_{V} \Pi_{\mu \alpha}^{V}\left(p_{1}\right)^{i i i i}\right\} \times\left\{g_{\nu \beta}-g_{V} \Pi_{\nu \beta}^{V}\left(p_{2}\right)^{i i i i}\right\} \\
& \times\left\{1+g_{S} \Pi_{S i i}\left(q^{2}\right)\right\} \bar{\Pi}_{\alpha \beta}^{S V V}\left(p_{1}, p_{2}\right)^{i i i}
\end{aligned}
$$

In the equal mass case both the full and the one-loop three-point function are fully transverse.

The one-loop two-point functions expanded to second order in the momenta is fully determined from the Ward Identities via

$$
\begin{aligned}
\bar{\Pi}_{\mu \nu}^{S V V}\left(p_{1}, p_{2}\right)^{i j k} & =\bar{\Pi}_{1}^{S V V i j k} g_{\mu \nu}+\bar{\Pi}_{2}^{S V V i j k}\left(p_{2 \mu} p_{1 \nu}-p_{1} \cdot p_{2} g_{\mu \nu}\right), \\
\bar{\Pi}_{1}^{S V V i j k} & =\frac{1}{M_{j}-M_{i}}\left\{\left(M_{i}-M_{k}\right) \bar{\Pi}_{S i k}^{M}-\left(M_{j}-M_{k}\right) \bar{\Pi}_{S j k}^{M}\right\}, \\
\bar{\Pi}_{2}^{S V V i j k} & =\frac{\bar{\Pi}_{V i k}^{(0+1)}-\bar{\Pi}_{V j k}^{(0+1)}}{M_{j}-M_{i}} .
\end{aligned}
$$

In the chiral limit these expressions reduce to

$$
\begin{aligned}
& \bar{\Pi}_{1}^{S V V \chi}=0, \\
& \bar{\Pi}_{2}^{S V V \chi}=-\frac{\bar{\Pi}_{V}^{(0+1) I}}{1-g_{S} \Delta} .
\end{aligned}
$$


The expression for the chiral limit full three-point functions is very simple

$$
\Pi_{\mu \nu}^{S V V}\left(p_{1}, p_{2}\right)^{\chi}=A \frac{m_{S}^{2}}{m_{S}^{2}-q^{2}} \frac{m_{V}^{2}}{m_{V}^{2}-p_{1}^{2}} \frac{m_{V}^{2}}{m_{V}^{2}-p_{2}^{2}}\left(p_{2 \mu} p_{1 \nu}-p_{1} \cdot p_{2} g_{\mu \nu}\right) .
$$

with

$$
A=-\bar{\Pi}_{V}^{(0+1) I}=-\frac{\langle\bar{q} q\rangle_{\chi}}{m_{V}^{4}} .
$$

This also satisfies the QCD short-distance requirement

$$
\lim _{\lambda \rightarrow \infty} \Pi_{\mu \nu}^{S V V}\left(\lambda p_{1}, \lambda p_{2}\right)^{\chi}=0 .
$$

\subsection{The Pseudoscalar-Vector-Axial-vector Three-Point Function}

This three-point functions has been studied in a related way in Ref. [11. The expression for the full Pseudoscalar-Vector-Axial-vector three-point function in terms of the one-loop one and two-point functions is in the case of $m_{i}=m_{k}$ :

$$
\begin{aligned}
\Pi_{\mu \nu}^{P V A}\left(p_{1}, p_{2}\right)^{i j k}= & \left\{g^{\mu \beta}-g_{V} \Pi_{V i k}^{\mu \beta}\left(p_{1}\right)\right\} \\
& \times\left\{\left(1+g_{S} \Pi_{P i j}\left(q^{2}\right)\right)\left(g^{\nu \gamma}-g_{V} \Pi^{A \nu \gamma}\left(p_{2}\right)^{k j}\right) \bar{\Pi}_{\beta \gamma}^{P V A}\left(p_{1}, p_{2}\right)^{i j k}\right. \\
& +g_{V} \Pi_{P i j}^{M}\left(q^{2}\right)\left(g^{\nu \gamma}-g_{V} \Pi^{A \nu \gamma}\left(p_{2}\right)^{k j}\right) \\
& \times\left(-\left(M_{i}+M_{j}\right) \bar{\Pi}_{\beta \gamma}^{P V A}\left(p_{1}, p_{2}\right)^{i j k}-i \bar{\Pi}_{\beta \gamma}^{V}\left(p_{1}\right)^{i k}+i \bar{\Pi}_{\beta \gamma}^{A}\left(p_{2}\right)^{j k}\right) \\
& +\left(1+g_{S} \Pi_{P i j}\left(q^{2}\right)\right) g_{S} i p_{2}^{\nu} \Pi_{P k j}^{M}\left(p_{2}^{2}\right) \bar{\Pi}_{\beta}^{P V P}\left(p_{1}, p_{2}\right)^{i j k} \\
& +g_{S} g_{V} i p_{2}^{\nu} \Pi_{P i j}^{M}\left(q^{2}\right) \Pi_{P k j}^{M}\left(p_{2}^{2}\right) \\
& \left.\times\left(-\left(M_{i}+M_{j}\right) \bar{\Pi}_{\beta}^{P V P}\left(p_{1}, p_{2}\right)^{i j k}+\bar{\Pi}_{\beta}^{S}\left(p_{1}\right)^{i k}-i \bar{\Pi}_{\beta}^{P}\left(p_{2}\right)_{j k}\right)\right\},
\end{aligned}
$$

where we have used the Ward identities

$$
\begin{aligned}
-i q^{\alpha} \bar{\Pi}_{\alpha \beta \gamma}^{A V A}\left(p_{1}, p_{2}\right)^{i j k} & =-\left(M_{i}+M_{j}\right) \bar{\Pi}_{\beta \gamma}^{P V A}\left(p_{1}, p_{2}\right)^{i j k}-i \bar{\Pi}_{\beta \gamma}^{V}\left(p_{1}\right)^{i k}+i \bar{\Pi}_{\beta \gamma}^{A}\left(p_{2}\right)^{j k}, \\
-i q^{\alpha} \bar{\Pi}_{\alpha \beta}^{A V P}\left(p_{1}, p_{2}\right)^{i j k} & =-\left(M_{i}+M_{j}\right) \bar{\Pi}_{\beta}^{P V P}\left(p_{1}, p_{2}\right)^{i j k}+\bar{\Pi}_{\beta}^{S}\left(p_{1}\right)^{i k}-i \bar{\Pi}_{\beta}^{P}\left(p_{2}\right)^{j k} .
\end{aligned}
$$

The one-loop three-point function up to second order in the momenta is determined fully from the one-loop Ward Identities.

$$
\begin{aligned}
\bar{\Pi}_{\mu \nu}^{P V A}\left(p_{1}, p_{2}\right)^{i j k}= & \bar{\Pi}_{1}^{P V A i j k} g_{\mu \nu}+\bar{\Pi}_{2}^{P V A i j k}\left(p_{2 \mu} p_{1 \nu}-p_{1} \cdot p_{2} g_{\mu \nu}\right) \\
& +C_{i j k}^{P V A}\left(q \cdot p_{1} g_{\mu \nu}-p_{1 \mu} p_{1 \nu}-p_{2 \mu} p_{1 \nu}\right)
\end{aligned}
$$

with

$$
\begin{aligned}
\bar{\Pi}_{1}^{P V A i j k} & =\frac{i}{M_{i}+M_{j}}\left\{\left(M_{j}+M_{k}\right) \bar{\Pi}_{P j k}^{M}-\left(M_{i}-M_{k}\right) \bar{\Pi}_{S i k}^{M}\right\}, \\
\bar{\Pi}_{2}^{P V A i j k} & =\frac{i}{M_{i}+M_{j}}\left(\bar{\Pi}_{V i k}^{(0+1)}-\bar{\Pi}_{A j k}^{(0+1)}\right), \\
C_{i j k}^{P V A} & =i\left(M_{j}+M_{k}\right) C_{k i j}^{V P P} .
\end{aligned}
$$


This expression can be worked out in the chiral limit using the values obtained earlier and compared with the chiral limit ChPT expression for this amplitude (see e.g. Ref. [11]).

$$
\begin{aligned}
\Pi_{P V A}^{\mu \nu C h P T}= & 2 i\langle\bar{q} q\rangle_{\chi}\left\{\left[\frac{\left(p_{1}+2 p_{2}\right)^{\mu} p_{2}^{\nu}}{p_{2}^{2} q^{2}}-\frac{g^{\mu \nu}}{q^{2}}\right]\right. \\
& +\left(p_{2}^{\mu} p_{1}^{\nu}-\frac{1}{2}\left(q^{2}-p_{1}^{2}-p_{2}^{2}\right) g^{\mu \nu}\right) \frac{4}{F_{0}^{2} q^{2}}\left(L_{9}+L_{10}\right) \\
& +\left(p_{1}^{2} p_{2}^{\mu} p_{2}^{\nu}+p_{2}^{2} p_{1}^{\mu} p_{1}^{\nu}-\frac{1}{2}\left(q^{2}-p_{1}^{2}-p_{2}^{2}\right) p_{1}^{\mu} p_{2}^{\nu}-p_{1}^{2} p_{2}^{2} g^{\mu \nu}\right) \frac{4}{F_{0}^{2} p_{2}^{2} q^{2}} L_{9}
\end{aligned}
$$

and leads to values of $L_{9}$ compatible with those obtained in Eq. (52) and

$$
L_{10}=-\frac{1}{2} F_{0}^{4} g_{V} \bar{\Pi}_{V}^{(0+1) \chi} \frac{\left(g_{V} g_{S}\langle\bar{q} q\rangle_{\chi} \bar{\Pi}_{P}^{M \chi}-1\right)}{g_{S}\langle\bar{q} q\rangle_{\chi} \bar{\Pi}_{P}^{M \chi}},
$$

which is the same as Eq. (22).

The three-point function in the chiral limit has a simple expression of the form

$$
\begin{aligned}
\Pi_{\mu \nu}^{P V A}\left(p_{1}, p_{2}\right)^{\chi}= & -\frac{2 i\langle\bar{q} q\rangle_{\chi}}{\left(p_{1}^{2}-m_{V}^{2}\right) q^{2}}\left\{\frac{P_{\mu \nu}\left(p_{1}, p_{2}\right)\left(m_{A}^{2}-m_{V}^{2}\right)+Q_{\mu \nu}\left(p_{1}, p_{2}\right)}{p_{2}^{2}-m_{A}^{2}}-\frac{2 Q_{\mu \nu}\left(p_{1}, p_{2}\right)}{p_{2}^{2}}\right\} \\
& +\frac{-2 i\langle\bar{q} q\rangle_{\chi}}{p_{2}^{2} q^{2}}\left(p_{1 \mu} p_{2 \nu}+2 p_{2 \mu} p_{2 \nu}-p_{2}^{2} g_{\mu \nu}\right) .
\end{aligned}
$$

The tensors $P_{\mu \nu}$ and $Q_{\mu \nu}$ are transverse and defined by

$$
\begin{aligned}
& P_{\mu \nu}\left(p_{1}, p_{2}\right)=p_{2 \mu} p_{1 \nu}-p_{1} \cdot p_{2} g_{\mu \nu} \\
& Q_{\mu \nu}\left(p_{1}, p_{2}\right)=p_{1}^{2} p_{2 \mu} p_{2 \nu}+p_{2}^{2} p_{1 \mu} p_{1 \nu}-p_{1} \cdot p_{2} p_{1 \mu} p_{2 \nu}-p_{1}^{2} p_{2}^{2} g_{\mu \nu} .
\end{aligned}
$$

By construction, this function satisfies the chiral Ward identities (see e.g. [11)

$$
\begin{aligned}
& p_{1 \mu} \Pi_{P V A}^{\mu \nu}\left(p_{1}, p_{2}\right)=-2 i\langle\bar{q} q\rangle_{\chi}\left[\frac{p_{2}^{\nu}}{p_{2}^{2}}-\frac{q^{\nu}}{q^{2}}\right] \\
& p_{2 \nu} \Pi_{P V A}^{\mu \nu}\left(p_{1}, p_{2}\right)=-2 i\langle\bar{q} q\rangle_{\chi} \frac{q^{\mu}}{q^{2}}
\end{aligned}
$$

that are the same as those involving the one-loop function $\bar{\Pi}_{P V A}^{\mu \nu}$ but replacing the constituent masses by current quark masses. The QCD short-distance relation

$$
\lim _{\lambda \rightarrow \infty} \Pi_{\mu \nu}^{P V A}\left(\lambda p_{1}, \lambda p_{2}\right)^{\chi}=0 .
$$

is also obeyed. 


\subsection{The Pseudo-scalar-Axial-vector-Scalar Three-Point Function}

Another order parameter is the sum of the Pseudoscalar-Axial-vector-Scalar and ScalarAxial-vector-Pseudoscalar three-point functions. These functions can be written in terms of the corresponding one-loop functions and the two-point functions following the same method as in the other sections

For the simpler case $m_{j}=m_{k}$

$$
\begin{aligned}
\Pi_{\mu}^{P A S}\left(p_{1}, p_{2}\right)^{i j k}= & \left\{1+g_{S} \Pi_{S j k}\left(p_{2}^{2}\right)\right\} \\
& \times\left\{\bar{\Pi}^{P A S \gamma}\left(p_{1}, p_{2}\right)^{i j k}\left(1+g_{S} \Pi_{P i j}\left(q^{2}\right)\right)\left(g_{\mu \gamma}-g_{V} \Pi_{\mu \gamma}^{A}\left(p_{1}\right)^{k i}\right)\right. \\
& +\bar{\Pi}^{A A S \alpha \gamma}\left(p_{1}, p_{2}\right)^{i j k}\left(-g_{V} i q_{\alpha} \Pi_{P}^{M i j}\left(q^{2}\right)\right)\left(g_{\mu \gamma}-g_{V} \Pi_{\mu \gamma}^{A}\left(p_{1}\right)^{k i}\right) \\
& +\bar{\Pi}^{P P S}\left(p_{1}, p_{2}\right)^{i j k}\left(1+g_{S} \Pi_{P i j}\left(q^{2}\right)\right)\left(g_{S} i p_{1 \mu} \Pi_{P k i}^{M}\left(p_{1}^{2}\right)\right) \\
& \left.+\bar{\Pi}_{\alpha}^{A P S}\left(p_{1}, p_{2}\right)^{i j k}\left(-g_{V} i q^{\alpha} \Pi_{P}^{M i j}\left(q^{2}\right)\right)\left(g_{S} i p_{1 \mu} \Pi_{P k i}^{M}\left(p_{1}^{2}\right)\right)\right\}
\end{aligned}
$$

and for the case $m_{i}=m_{j}$

$$
\begin{aligned}
\Pi_{\mu}^{S A P}\left(p_{1}, p_{2}\right)^{i j k}= & \left\{1+g_{S} \Pi_{S i j}\left(q^{2}\right)\right\} \\
& \times\left\{\bar{\Pi}^{S A P \gamma}\left(p_{1}, p_{2}\right)^{i j k}\left(1+g_{S} \Pi_{P j k}\left(p_{2}^{2}\right)\right)\left(g_{\mu \gamma}-g_{V} \Pi_{\mu \gamma}^{A}\left(p_{1}\right)^{k i}\right)\right. \\
& +\bar{\Pi}^{S A A \alpha \gamma}\left(p_{1}, p_{2}\right)^{i j k}\left(g_{V} i p_{2 \alpha} \Pi_{P}^{M i j}\left(p_{2}^{2}\right)\right)\left(g_{\mu \gamma}-g_{V} \Pi_{\mu \gamma}^{A}\left(p_{1}\right)^{k i}\right) \\
& +\bar{\Pi}^{S P P}\left(p_{1}, p_{2}\right)^{i j k}\left(1+g_{S} \Pi_{P j k}\left(p_{2}^{2}\right)\right)\left(g_{S} i p_{1 \mu} \Pi_{P k i}^{M}\left(p_{1}^{2}\right)\right) \\
& +\bar{\Pi}^{S P A \alpha}\left(p_{1}, p_{2}\right)^{i j k}\left(g_{V} i p_{2 \alpha} \Pi_{P}^{M j k}\left(p_{2}^{2}\right)\right)\left(g_{S} i p_{1 \mu} \Pi_{P k i}^{M}\left(p_{1}^{2}\right)\right)
\end{aligned}
$$

The most general expressions for the one-loop three-point functions $\bar{\Pi}_{\gamma}^{S A P}\left(p_{1}, p_{2}\right)^{i j k}$ and $\bar{\Pi}_{\gamma}^{S A P}\left(p_{1}, p_{2}\right)^{i j k}$ up to order $O\left(p^{3}\right)$ and compatible with all the symmetries

$$
\begin{aligned}
& \bar{\Pi}_{\mu}^{P A S}\left(p_{1}, p_{2}\right)^{i j k}=p_{1 \mu} \bar{\Pi}_{1}^{P A S i j k}+p_{2 \mu} \bar{\Pi}_{2}^{P A S i j k}+C_{i j k}^{P A S}\left(p_{1}^{2} p_{2 \mu}-p_{1} \cdot p_{2} p_{1 \mu}\right) \\
& \bar{\Pi}_{\mu}^{S A P}\left(p_{1}, p_{2}\right)^{i j k}=p_{1 \mu} \bar{\Pi}_{1}^{S A P i j k}+p_{2 \mu} \bar{\Pi}_{2}^{S A P i j k}-C_{k j i}^{P A S}\left(p_{1}^{2} p_{2 \mu}-p_{1} \cdot p_{2} p_{1 \mu}\right)
\end{aligned}
$$

There is only one constant at order $O\left(p^{3}\right)$ that remains unknown when we apply all the symmetry criteria. The functions in the term of order $O(p)$ are fully determined by the use of the one-loop Ward identities

$$
\begin{aligned}
\bar{\Pi}_{1}^{P A S i j k} & =i \frac{\bar{\Pi}_{P i j}^{M}-\bar{\Pi}_{P i k}^{M}}{M_{j}-M_{k}} \\
\bar{\Pi}_{2}^{P A S i j k} & =i \frac{\bar{\Pi}_{S j k}^{M}+\bar{\Pi}_{P i k}^{M}}{M_{i}+M_{j}}+i \frac{\bar{\Pi}_{P i j}^{M}-\bar{\Pi}_{P i k}^{M}}{M_{j}-M_{k}} \\
\bar{\Pi}_{1}^{S A P i j k} & =i \frac{\bar{\Pi}_{S i j}^{M}-\bar{\Pi}_{P i k}^{M}}{M_{j}+M_{k}}
\end{aligned}
$$




$$
\bar{\Pi}_{2}^{S A P i j k}=i \frac{\bar{\Pi}_{S i j}^{M}-\bar{\Pi}_{P i k}^{M}}{M_{j}+M_{k}}+i \frac{\bar{\Pi}_{P j k}^{M}-\bar{\Pi}_{P i k}^{M}}{M_{i}-M_{j}}
$$

Using the values of the coupling constants $L_{5}$ and $L_{8}$ we obtained from two-point functions, the functions $\Pi_{\mu}^{P A S}\left(p_{1}, p_{2}\right)^{i j k}$ and $\Pi_{\mu}^{S A P}\left(p_{1}, p_{2}\right)^{i j k}$ have the correct behaviour at long distance as described by Chiral Perturbation Theory. In this limit the unknown constant $C_{i j k}^{P A S}$ is not involved.

The sum of the two three-point functions in the chiral limit can be written in a fairly simple fashion

$$
\begin{aligned}
\Pi_{\mu}^{P A S+S A P}\left(p_{1}, p_{2}\right)^{\chi}= & i B_{0}^{2} F_{0}^{2} \frac{m_{S}^{2}}{\left(m_{S}^{2}-q^{2}\right)\left(m_{S}^{2}-p_{2}^{2}\right)\left(m_{A}^{2}-p_{1}^{2}\right) p_{2}^{2} q^{2} p_{1}^{2}} \\
& \times\left\{p_{2 \mu} 4\left(m_{A}^{2}+D_{\chi}^{P A S} p_{1}^{2}\right) p_{1}^{2}\left(q^{2}-p_{2}^{2}\right)\right. \\
& +p_{1 \mu}\left[-2 m_{S}^{2}\left(q^{2}+p_{2}^{2}\right)\left(m_{A}^{2}-p_{1}^{2}\right)-2 m_{A}^{2}\left(p_{1}^{2}\left(p_{2}^{2}-q^{2}\right)-2 q^{2} p_{2}^{2}\right)\right. \\
& \left.\left.-2 p_{1}^{2}\left(p_{2}^{4}+q^{4}\right)-2 D_{\chi}^{P A S} p_{1}^{2}\left(q^{2}-p_{1}^{2}-p_{2}^{2}\right)\left(q^{2}-p_{2}^{2}\right)\right]\right\}
\end{aligned}
$$

with

$$
D_{\chi}^{P A S}=i C_{\chi}^{P A S} \frac{g_{S}\langle\bar{q} q\rangle_{\chi}}{g_{V} \bar{\Pi}_{P}^{M \chi} \bar{\Pi}_{V}^{(0+1) \chi}} .
$$

\section{Comparison with experiment}

The input we use for $\langle\bar{q} q\rangle_{\chi}$ is the value derived from sum rules in Ref. [19, which is in agreement with most recent sum rules determinations of this condensate and of light quark masses -see [20] for instance- and the lattice light quark masses world average in [21]. The value of $F_{0}$ is from Ref. [22] and the remaining masses are those from the PDG.

$$
\begin{aligned}
F_{0}=(0.087 \pm 0.006) \mathrm{GeV}, & m_{V}=0.770 \mathrm{GeV}, \\
m_{A}=1.230 \mathrm{GeV}, & m_{S}=0.985 \mathrm{GeV}, \\
\frac{\langle\bar{u} u+\bar{d} d\rangle^{\overline{M S}}\left(m_{V}\right)}{2}= & \langle\bar{q} q\rangle_{\chi}^{\overline{M S}}\left(m_{V}\right)=-(0.0091 \pm 0.0020) \mathrm{GeV}^{3} .
\end{aligned}
$$

Putting in the various relations, we immediately obtain

$$
\begin{aligned}
f_{V} & =0.15[0.20] \text { [4, 6], } \\
f_{A} & =0.057[0.097 \pm 0.022][4,[6], \\
L_{5}\left(m_{V}\right) & =1.95 \cdot 10^{-3}\left[(1.0 \pm 0.2) \cdot 10^{-3}\right][22], \\
L_{8}\left(m_{V}\right) & =0.5 \cdot 10^{-3}\left[(0.6 \pm 0.2) \cdot 10^{-3}\right][22], \\
L_{9}\left(m_{V}\right) & =6.8 \cdot 10^{-3}\left[(5.93 \pm 0.43] \cdot 10^{-3}\right][23], \\
L_{10}\left(m_{V}\right) & =-4.4 \cdot 10^{-3}\left[(-4.4 \pm 0.7) \cdot 10^{-3}\right][23,24] .
\end{aligned}
$$


These numbers ${ }^{5}$ are in reasonable agreement with the experimental values given in brackets with the possible exception of $L_{5}$ which is rather high. We expect to have an uncertainty between $30 \%$ and $40 \%$ in our hadronic predictions. The values in Eq. (79) do not depend on the value of the quark condensate.

We cannot determine $\Delta$ at this level. The three-point functions $P S P, V P P, S V V$ and $P V A$ can be rewritten in terms of the above inputs. There is more freedom in those functions by expanding the underlying $\bar{\Pi}$ functions to higher order. These extra terms can usually be determined from the short-distance constraints up to the problem discussed in Sect. 6.

\section{Difficulties in Going Beyond the One-Resonance Approximation}

An obvious question to ask is whether we can easily go beyond the one resonance per channel approximation used above using the general resummation based scheme. At first sight one would have said that this can be done simply by including higher powers in the expansion of the one-loop two-point functions and/or giving $g_{S}, g_{V}$ a momentum dependence. Since we want to keep the nice analytic behaviour expected in the large $N_{c}$ limit with only poles and have simple expressions for the one-loop functions and $g_{S}, g_{V}$, it turns out to be very difficult to accomplish. We have tried many variations but essentially the same type of problems always showed up, related to the fact that the coefficients of poles of two-point functions obey positivity constraints. Let us concentrate on the scalar two-point function in the chiral limit to illustrate the general problem.

In this limit the full two-point function can be written in terms of the one-loop function as

$$
\Pi_{S}\left(q^{2}\right)=\frac{\bar{\Pi}_{S}\left(q^{2}\right)}{1-g_{S} \bar{\Pi}_{S}\left(q^{2}\right)} .
$$

If we want to give $g_{S}$ a polynomial dependence on $q^{2}$ this two-point function generally becomes far too convergent in the large $q^{2}$ limit. The other way to introduce more poles is to expand $\bar{\Pi}\left(q^{2}\right)$ beyond what we have done before to quartic or higher order. For the case of two-poles this means we want

$$
1-g_{S} \bar{\Pi}_{S}\left(q^{2}\right)=a\left(q^{2}-m_{1}^{2}\right)\left(q^{2}-m_{2}^{2}\right) .
$$

However that means we can rewrite

$$
\Pi_{S}\left(q^{2}\right)=-\frac{1}{g_{S}}+\frac{1}{g_{S} a\left(m_{1}^{2}-m_{2}^{2}\right)}\left(\frac{1}{q^{2}-m_{1}^{2}}-\frac{1}{q^{2}-m_{2}^{2}}\right) .
$$

From Eq. (82) it is obvious that the residues of the two poles will have opposite signs, thus preventing this simple approach for including more resonances. We have illustrated

\footnotetext{
${ }^{5}$ The value for $L_{10}$ used the values of $L_{9}$ from [23, the $2 l_{5}-l_{6}$ value from 24] and the $p^{4}$ relation $2 l_{5}-l_{6}=2 L_{9}+2 L_{10}$.
} 
the problem here for the simplest extensions but it persists as long as both $g_{S}, g_{V}$ and the one-loop two-point functions are fairly smooth functions.

\section{A General Problem in Short-Distance Constraints in Higher Green Functions}

At this level we have expanded our one-loop two-point functions to at most second nontrivial order in the momenta and we found that it was relatively easy to satisfy the shortdistance constraints involving exact zeros. However, if we check the short-distance relations for the three-point functions that are order parameters given in Eqs. (A.1), (A.2) and (A.3) and compare with short-distance properties of our model three-point functions of (44), (58) and (67), we find that they are typically too convergent. In this subsection we will discuss how this cannot be remedied in general without spoiling the parts we have already matched. In fact, we will show how in general this cannot be done using a single or any finite number of resonances per channel type of approximations. An earlier example where single resonance does not allow to reproduce all short-distance constraints was found in Ref. [11].

First look at the function $\Pi^{P S P}$ and see whether by adding terms in the expansion in $q^{2}, p_{1}^{2}, p_{2}^{2}$ to $\bar{\Pi}^{P S P}\left(p_{1}, p_{2}\right)^{\chi}$ beyond those considered in Eq. (38) we can satisfy the shortdistance requirement of Eq. (A.1). It can be easily seen that setting

$$
\begin{aligned}
\bar{\Pi}^{P S P}\left(p_{1}, p_{2}\right)^{\chi} & =\left.\bar{\Pi}^{P S P}\left(p_{1}, p_{2}\right)^{\chi}\right|_{\mathrm{Eq} .(138)}+\bar{\Pi}_{5}^{P S P \chi}\left(q^{4}+p_{2}^{4}-2 q^{2} p_{2}^{2}-p_{1}^{4}\right) \\
\bar{\Pi}_{5}^{P S P \chi} & =\frac{\left(\bar{\Pi}_{P}^{M \chi}\right)^{3}}{16\langle\bar{q} q\rangle_{\chi}^{2}\left(1-2 g_{S} g_{V}\langle\bar{q} q\rangle_{\chi} \bar{\Pi}_{P}^{M \chi}\right)}
\end{aligned}
$$

makes the short-distance constraint Eq. (A.1) satisfied. However, a problem is that now we obtain a very bad short-distance behaviour for the pion scalar form factor $F_{S}^{\chi}\left(p_{1}^{2}\right)$ which diverges as $p_{1}^{2}$ rather than going to zero. Inspection of the mechanism behind this shows that this is a general problem going beyond the single three-point function and model discussed here.

The problem is more generally a problem between the short-distance requirements on form factors and cross-sections, many of which can be qualitatively derived from the quarkcounting rules or more quantitatively using the methods of Ref. [17, with the short-distance properties of general Green functions.

The quark-counting rules typically require a form factor, here $F_{S}^{\chi}\left(p_{1}^{2}\right)$, to vanish as $1 / p_{1}^{2}$ for large $p_{1}^{2}$. The presence of the short-distance part proportional to $p_{1}^{2} /\left(q^{2} p_{2}^{2}\right)$ in the short distance expansion of $\Pi^{P S P}\left(p_{1}, p_{2}\right)^{\chi}$ then requires a coupling of the hadron in the $P$ channel to the $S$ current proportional to $p_{1}^{2}$ (or via a coupling to a hadron in the $S$ channel which in turn couples to the $S$ current, this complication does not invalidate the argument below). In the general class of models with hadrons coupling with point-like couplings the negative 
powers in Green functions can only be produced by a hadron propagator. The positive power present in the short-distance expression must thus be present in the couplings of the hadrons. This in turn implies that this power is present in the form factor of at least some hadrons. The latter is forbidden by the quark-counting rule.

It is clear that for at most a single resonance in each channel there is no solution to this set of constraints. In fact, as will show below, there is no solution to this problem for any finite number of resonances in any channel. This shows that even for order parameters the approach of saturation by resonances might have to be supplemented by a type of continuum. We will illustrate the problem for the PSP three-point function. The general expression, labeling resonances in the first $P$-channel by $i$, in the $S$-channel by $j$ and in the last $P$-channel by $k$ is

$$
\begin{aligned}
\Pi^{P S P}\left(p_{1}, p_{2}\right)^{\chi}= & f_{0}\left(q^{2}, p_{1}^{2}, p_{2}^{2}\right)+\sum_{i} \frac{f_{1 i}\left(p_{1}^{2}, p_{2}^{2}\right)}{\left(q^{2}-m_{i}^{2}\right)}+\sum_{j} \frac{f_{2 j}\left(q^{2}, p_{2}^{2}\right)}{\left(p_{1}^{2}-m_{j}^{2}\right)}+\sum_{k} \frac{f_{3 k}\left(q^{2}, p_{2}^{2}\right)}{\left(p_{2}^{2}-m_{k}^{2}\right)} \\
& +\sum_{i j} \frac{f_{4 i j}\left(p_{2}^{2}\right)}{\left(q^{2}-m_{i}^{2}\right)\left(p_{1}^{2}-m_{j}^{2}\right)}+\sum_{i k} \frac{f_{5 i k}\left(p_{1}^{2}\right)}{\left(q^{2}-m_{i}^{2}\right)\left(p_{2}^{2}-m_{k}^{2}\right)} \\
& +\sum_{j k} \frac{f_{6 j k}\left(q^{2}\right)}{\left(p_{1}^{2}-m_{j}^{2}\right)\left(p_{2}^{2}-m_{k}^{2}\right)}+\sum_{i j k} \frac{f_{i j k}}{\left(q^{2}-m_{i}^{2}\right)\left(p_{1}^{2}-m_{j}^{2}\right)\left(p_{2}^{2}-m_{k}^{2}\right)}
\end{aligned}
$$

The couplings $f_{i}$ are polynomials in their respective arguments. The short-distance constraint now requires $f_{0}\left(q^{2}, p_{1}^{2}, p_{2}^{2}\right)=0$ and various cancellations between coefficients of the other functions. The presence of the term $p_{1}^{2} /\left(q^{2} p_{2}^{2}\right)$ now requires the presence of at least a nonzero term of order $p_{1}^{2}$ in one of the $f_{5 i k}\left(p_{1}^{2}\right)$. However the Green function can then be used to extract the scalar (transition) form factor between hadron $i$ and $k$ which necessarily increases as $p_{1}^{2}$ which is forbidden by the quark-counting rules for this (transition) scalar form factor. The terms with $p_{2}^{2} /\left(q^{2} p_{1}^{2}\right)$ and $q^{2} /\left(p_{1}^{2} p_{2}^{2}\right)$ obviously leads to similar problems but in other (transition) form factors.

We have discussed the problem here for one specific three-point function but it is clear that this is a more general problem for three-point functions. For Green function with more than three insertions similar conflicts with the quark counting rules will probably arise also from hadron-hadron scattering amplitudes.

\section{Conclusions}

In this paper we have constructed a new approximation to low and intermediate energy hadronic quantities. Our approach naturally fits in the large $N_{c}$ limit and incorporates chiral symmetry constraints by construction. We have shown that many short-distance constraints can be easily incorporated but pointed out that our model, but also a more general saturation by hadrons approach, cannot reconcile all short-distance constraints due to a general conflict between short distance constraints on Green functions and those on form factors and cross-sections that can be obtained from those Green functions via LSZ reduction. 
We have also shown how our approach incorporates the gap equation and the concept of a constituent quark mass following directly from the Ward Identities and the resummation assumption.

We have also compared our results with experimental results for hadronic observables and found reasonable agreement.

\section{Acknowledgements}

E.G. is indebted to MECD (Spain) for a F.P.U. Fellowship. This research is supported in part by the Swedish Research Council, by MCYT (Spain) Grant No. FPA2000-1558 (E.G and J.P.) and by Junta de Andalucía Grant No. FQM-101 (E.G. and J.P.). E.G. and J.P. thank Lund University for hospitality.

\section{A Some Short-Distance Relations Beyond those Men- tioned in the Text}

We have calculated or recalculated several short-distance behaviours of three-point functions.

$$
\begin{gathered}
\lim _{\lambda \rightarrow \infty} \Pi^{P S P}\left(\lambda p_{1}, \lambda p_{2}\right)^{\chi}=\frac{\langle\bar{q} q\rangle_{\chi}}{2 \lambda^{2}}\left\{\frac{p_{2}^{2}}{q^{2} p_{1}^{2}}+\frac{q^{2}}{p_{1}^{2} p_{2}^{2}}-\frac{p_{1}^{2}}{q^{2} p_{2}^{2}}-\frac{2}{p_{1}^{2}}\right\} \\
\lim _{\lambda \rightarrow \infty} \Pi^{S V V}\left(\lambda p_{1}, \lambda p_{2}\right)^{\chi}=\frac{\langle\bar{q} q\rangle_{\chi}}{2 \lambda^{2} q^{2} p_{1}^{2} p_{2}^{2}}\left\{-4 p_{2}^{2} p_{1 \mu} p_{1 \nu}-2\left(p_{1}^{2}+p_{2}^{2}-q^{2}\right) p_{1 \mu} p_{2 \nu}\right. \\
\left.-2\left(p_{1}^{2}+p_{2}^{2}+q^{2}\right) p_{2 \mu} p_{1 \nu}-4 p_{1}^{2} p_{2 \mu} p_{2 \nu}+\left(q^{4}-\left(p_{1}^{2}-p_{2}^{2}\right)^{2}\right) g_{\mu \nu}\right\} \\
\lim _{\lambda \rightarrow \infty} \Pi^{P V A}\left(\lambda p_{1}, \lambda p_{2}\right)^{\chi}=\frac{i\langle\bar{q} q\rangle_{\chi}}{2 \lambda^{2} q^{2} p_{1}^{2} p_{2}^{2}}\left\{4 p_{2}^{2} p_{1 \mu} p_{1 \nu}-2\left(q^{2}+p_{1}^{2}-p_{2}^{2}\right) p_{1 \mu} p_{2 \nu}\right. \\
\left.+2\left(q^{2}+p_{2}^{2}-p_{1}^{2}\right) p_{2 \mu} p_{1 \nu}-4 p_{1}^{2} p_{2 \mu} p_{2 \nu}+\left(p_{2}^{4}-\left(p_{1}^{2}-q^{2}\right)^{2}\right) g_{\mu \nu}\right\}
\end{gathered}
$$

Some of these have been mentioned in Refs. [11, 12.

The following are to our knowledge new:

$$
\begin{aligned}
\lim _{\lambda \rightarrow \infty} & \left(\Pi^{P A S}\left(\lambda p_{1}, \lambda p_{2}\right)^{\chi}+\Pi^{S A P}\left(\lambda p_{1}, \lambda p_{2}\right)^{\chi}\right)=i 4 \pi \alpha_{S} \frac{N_{c}^{2}-1}{N_{c}^{2}} \frac{\langle\bar{q} q\rangle_{\chi}^{2}}{\lambda^{5}} \\
& \times\left\{\frac{2 p_{1 \mu}}{p_{1}^{2}}\left(\frac{1}{q^{4}}+\frac{1}{p_{2}^{4}}+\frac{1}{p_{2}^{2} q^{2}}\right)+\frac{p_{2 \mu}}{p_{2}^{2}}\left(\frac{-1}{q^{4}}+\frac{1}{p_{2}^{2} q^{2}}\right)+\frac{q_{\mu}}{q^{2}}\left(\frac{1}{p_{2}^{4}}-\frac{1}{p_{2}^{2} q^{2}}\right)\right\}
\end{aligned}
$$




$$
\begin{aligned}
\lim _{\lambda \rightarrow \infty} \lim _{m_{q} \rightarrow 0} \frac{\partial}{\partial m_{i}}\left(\Pi^{P A S}\left(\lambda p_{1}, \lambda p_{2}\right)^{i j k}+\Pi^{S A P}\left(\lambda p_{1}, \lambda p_{2}\right)^{i j k}\right) & =-2 i\langle\bar{q} q\rangle_{\chi} \frac{p_{2 \mu}}{\lambda^{3} p_{2}^{2} q^{2}}, \\
\lim _{\lambda \rightarrow \infty} \lim _{m_{q} \rightarrow 0} \frac{\partial}{\partial m_{j}}\left(\Pi^{P A S}\left(\lambda p_{1}, \lambda p_{2}\right)^{i j k}+\Pi^{S A P}\left(\lambda p_{1}, \lambda p_{2}\right)^{i j k}\right) & =2 i\langle\bar{q} q\rangle_{\chi} \frac{p_{1 \mu}}{\lambda^{3} p_{1}^{2}}\left(\frac{1}{q^{2}}+\frac{1}{p_{2}^{2}}\right), \\
\lim _{\lambda \rightarrow \infty} \lim _{m_{q} \rightarrow 0} \frac{\partial}{\partial m_{k}}\left(\Pi^{P A S}\left(\lambda p_{1}, \lambda p_{2}\right)^{i j k}+\Pi^{S A P}\left(\lambda p_{1}, \lambda p_{2}\right)^{i j k}\right) & =2 i\langle\bar{q} q\rangle_{\chi} \frac{q_{\mu}}{\lambda^{3} p_{2}^{2} q^{2}} .
\end{aligned}
$$

\section{References}

[1] G. 't Hooft, Nucl. Phys. B 72 (1974) 461;

A. V. Manohar, hep-ph/9802419, Les Houches Summer School in Theoretical Physics, Session 68: Probing the Standard Model of Particle Interactions, Les Houches, France, 28 Jul - 5 Sep 1997 F. David and R. Gupta eds.

[2] Y. Nambu and G. Jona-Lasinio, Phys. Rev. 122 (1961) 345.

[3] T. Hatsuda and T. Kunihiro, Phys. Rept. 247 (1994) 221 hep-ph/9401310;

S. P. Klevansky, Rev. Mod. Phys. 64 (1992) 649.

[4] J. Bijnens, Phys. Rept. 265 (1996) 369 hep-ph/9502335.

[5] A. Manohar and H. Georgi, Nucl. Phys. B 234 (1984) 189;

D. Espriu, E. de Rafael and J. Taron, Nucl. Phys. B 345 (1990) 22 [Erratum-ibid. B 355 (1991) 278].

[6] J. Bijnens, C. Bruno and E. de Rafael, Nucl. Phys. B 390 (1993) 501 hep-ph/9206236.

[7] J. Bijnens, E. de Rafael and H. -q. Zheng, Z. Phys. C 62 (1994) 437 hep-ph/9306323.

[8] J. Bijnens and J. Prades, Z. Phys. C 64 (1994) 475 hep-ph/9403233.

[9] S. Peris, M. Perrottet and E. de Rafael, J. High Energy Phys. 05 (1998) 011 hep-ph/9805442;

M. Knecht, S. Peris and E. de Rafael, Nucl. Phys. Proc. Suppl. 86 (2000) 279 hep-ph/9910396.

[10] M. F. Golterman and S. Peris, Phys. Rev. D 61 (2000) 034018 hep-ph/9908252.

[11] M. Knecht and A. Nyffeler, Eur. Phys. J. C 21 (2001) 659 hep-ph/0106034.

[12] B. Moussallam and J. Stern, hep-ph/9404353.

B. Moussallam, Phys. Rev. D 51 (1995) 4939 hep-ph/9407402; Nucl. Phys. B 504 (1997) 381 hep-ph/9701400. 
[13] J. Bijnens, E. Gámiz and J. Prades, J. High Energy Phys. 10 (2001) 009 hep-ph/0108240.

J. Bijnens and J. Prades, J. High Energy Phys. 06 (2000) 035 hep-ph/0005189; J. High Energy Phys. 01 (2000) 002 hep-ph/9911392; J. High Energy Phys. 01 (1999) 023 hep-ph/9811472; Nucl. Phys. B 490 (1997) 239 hep-ph/9610360.

J. Bijnens, E. Pallante and J. Prades, Nucl. Phys. B 521 (1998) 305 hep-ph/9801326.

J. Bijnens, A. Fayyazuddin and J. Prades, Phys. Lett. B 379 (1996) 209 hep-ph/9512374.

[14] J. Bijnens, E. Pallante and J. Prades, Nucl. Phys. B 626 (2002) 410 hep-ph/0112255; Nucl. Phys. B 474 (1996) 379 hep-ph/9511388|; Phys. Rev. Lett. 75 (1995) 1447 [Erratum-ibid. 75 (1995) 3781] hep-ph/9505251.

J. Bijnens and J. Prades, Nucl. Phys. B 444 (1995) 523 hep-ph/9502363|; Phys. Lett. B 342 (1995) 331 hep-ph/9409255.

[15] S. Weinberg, Phys. Rev. Lett. 18 (1967) 507.

[16] M. A. Shifman, A. I. Vainshtein and V. I. Zakharov, Nucl. Phys. B 147 (1979) 385;

L. J. Reinders, H. Rubinstein and S. Yazaki, Phys. Rept. 127 (1985) 1.

[17] G. P. Lepage and S. J. Brodsky, Phys. Lett. B 87 (1979) 359.

G. P. Lepage and S. J. Brodsky, Phys. Rev. D 22 (1980) 2157.

[18] M. Jamin, J. A. Oller and A. Pich, Nucl. Phys. B 622 (2002) 279 hep-ph/0110193. A. Pich, hep-ph/0205030.

[19] J. Bijnens, J. Prades and E. de Rafael, Phys. Lett. B 348 (1995) 226 hep-ph/9411285;

K. Maltman and J. Kambor, Phys. Lett. B 517 (2001) 332 hep-ph/0107060.

[20] M. Jamin, J. A. Oller and A. Pich, Eur. Phys. J. C 24 (2002) 237 hep-ph/0110194.

[21] T. Kaneko, Nucl. Phys. Proc. Suppl. 106 (2002) 133 hep-lat/0111005.

[22] G. Amorós, J. Bijnens and P. Talavera, Nucl. Phys. B 602 (2001) 87 hep-ph/0101127.

[23] J. Bijnens and P. Talavera, J. High Energy Phys. 03 (2002) 046 hep-ph/0203049.

[24] J. Bijnens and P. Talavera, Nucl. Phys. B 489 (1997) 387 hep-ph/9610269. 